\title{
Leveraging Nonhuman Primate Multisensory Neurons and Circuits in Assessing Consciousness Theory
}

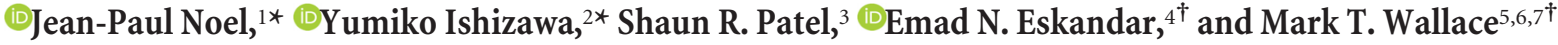 \\ ${ }^{1}$ Center for Neural Science, New York University, New York, New York 10003, Departments of ${ }^{2}$ Anesthesia, Critical Care, \& Pain Medicine, ${ }^{3}$ Neurology, \\ Massachusetts General Hospital, Harvard Medical School, Boston, Massachusetts 02114, ${ }^{4}$ Leo M. Davidoff Department of Neurological Surgery, Albert \\ Einstein College of Medicine, Bronx, New York 10461, ${ }^{5}$ Department of Hearing and Speech, Vanderbilt University Medical School, Nashville, Tennessee \\ 37235, ${ }^{6}$ Department of Psychology, Vanderbilt University, Nashville, Tennessee 37235, and ${ }^{7}$ Department of Psychiatry and Behavioral Sciences, Vanderbilt \\ Medical School, Nashville, Tennessee 37235
}

Both the global neuronal workspace (GNW) and integrated information theory (IIT) posit that highly complex and interconnected networks engender perceptual awareness. GNW specifies that activity recruiting frontoparietal networks will elicit a subjective experience, whereas IIT is more concerned with the functional architecture of networks than with activity within it. Here, we argue that according to IIT mathematics, circuits converging on integrative versus convergent yet non-integrative neurons should support a greater degree of consciousness. We test this hypothesis by analyzing a dataset of neuronal responses collected simultaneously from primary somatosensory cortex (S1) and ventral premotor cortex (vPM) in nonhuman primates presented with auditory, tactile, and audio-tactile stimuli as they are progressively anesthetized with propofol. We first describe the multisensory (audio-tactile) characteristics of S1 and vPM neurons (mean and dispersion tendencies, as well as noise-correlations), and functionally label these neurons as convergent or integrative according to their spiking responses. Then, we characterize how these different pools of neurons behave as a function of consciousness. At odds with the IIT mathematics, results suggest that convergent neurons more readily exhibit properties of consciousness (neural complexity and noise correlation) and are more impacted during the loss of consciousness than integrative neurons. Last, we provide support for the GNW by showing that neural ignition (i.e., same trial coactivation of S1 and vPM) was more frequent in conscious than unconscious states. Overall, we contrast GNW and IIT within the same single-unit activity dataset, and support the GNW.

Key words: complexity; consciousness; integrated information; noise correlations; primary somatosensory cortex; ventral premotor

Significance Statement

A number of prominent theories of consciousness exist, and a number of these share strong commonalities, such as the central role they ascribe to integration. Despite the important and far reaching consequences developing a better understanding of consciousness promises to bring, for instance in diagnosing disorders of consciousness (e.g., coma, vegetative-state, locked-in syndrome), these theories are seldom tested via invasive techniques (with high signal-to-noise ratios), and never directly confronted within a single dataset. Here, we first derive concrete and testable predictions from the global neuronal workspace and integrated information theory of consciousness. Then, we put these to the test by functionally labeling specific neurons as either convergent or integrative nodes, and examining the response of these neurons during anesthetic-induced loss of consciousness.

\section{Introduction}

Understanding the neural architecture enabling arousal or wakefulness (i.e., level or state of consciousness) and conscious experience

\footnotetext{
Received April 24, 2019; revised June 27, 2019; accepted July 19, 2019.

Author contributions: J.-P.N. designed research; J.-P.N., Y.I., and S.R.P. performed research; J.-P.N. and S.R.P. analyzed data; E.N.E. contributed unpublished reagents/analytic tools; J.-P.N., Y.I., S.R.P., E.N.E., and M.T.W. wrote the paper.

This work was supported by F31MH112336 to J.-P.N. We thank Dr. Randolph Blake for insightful comments on an earlier version of the paper.

The authors declare no competing financial interests.

*J.P.-N. and Y.I. contributed equally as co-first authors.
}

(i.e., content of consciousness) remains a central unanswered question in systems neuroscience despite its profound clinical implications in coma, vegetative-state, minimal-consciousness, and general anesthesia (Laureys et al., 2004; Monti et al., 2010; Giacino et al., 2014). Although in recent years a number of electrophysiological

\footnotetext{
${ }^{\dagger}$ E.N.E. and M.T.W. contributed equally as co-senior authors.

Extended Data is available at https://www.jneurosci.org/content/39/38/7485/tab-figures-data\#figdata supplementary-materials.

Correspondence should be addressed to Jean-Paul Noel at Jpn5@nyu.edu.

https://doi.org/10.1523/JNEUROSCI.0934-19.2019

Copyright $\odot 2019$ the authors
} 
measures of consciousness/awareness have been proposed (Owen et al., 2006; Casali et al., 2013; Owen, 2014) these tend to be more practical than principled, and grounded more in the realm of engineering than neurobiology.

Lacking a mechanistic account of consciousness, a number of theorists and researchers have started from empirical observations or phenomenological axioms to derive consciousness theories. A number of these theories share many commonalities, as well as a number of practical and conceptual differences, as exemplified by the global neuronal workspace (GNW; Dehaene and Changeux, 2011; Dehaene et al., 2017) and integrated information theory (IIT; Tononi, 2012; Oizumi et al., 2014; Tononi et al., 2016; but see Lamme, 2006; Graziano and Kastner, 2011; Lau and Rosenthal, 2011; Block et al., 2014, for a number of other theories).

The GNW posits that an external stimulus will evoke a conscious experience if the associated neural information is widely distributed across distinct brain areas and networks; most prominently in the prefrontal cortex (Dehaene and Changeux, 2011). Rendering sensory information globally available results in a coherent neural assembly of sustained activity and is most readily indexed via "neural ignition", the nonlinear process whereby in unconscious states neuronal activation profiles remain encapsulated within their specialized subsystems, whereas in conscious experiences these activation patterns are widely distributed (for experimental evidence, see van Vugt et al., 2018; for a recent computational treatise, see Joglekar et al., 2018).

In a similar manner, IIT is a systems-level theory of consciousness also postulating that complex and highly interconnected neural networks support subjective experience (Tononi and Edelman, 1998; Tononi et al., 2016). In contrast to GNW, however, the mathematics developed in hand with the IIT (Oizumi et al., 2014) are argued to apply to all physical networks, and in turn IIT is arguably more focused on the architecture of networks rather than the activity within these. In more detail, Tononi and colleagues argue that each conscious experience is highly informative, as it represents a particular instance among a vast repertoire of potential experiences, and is highly unified, unable of being deconstructed into subexperiences that are each independently perceived. In turn, the IIT specifies that an organism may support conscious experience if imbued with an information processing architecture that is capable of supporting both high differentiation (i.e., large repertoire of possible states) and integration (i.e., strong statistical dependencies between system components).

Unfortunately, the neurophysiological data, particularly that focused at the microcircuit level (Mashour, 2018), that bears directly on these theories is limited, in particular the IIT given its computational overhead. Indeed, one strength of the IIT is that it explicitly generates a metric of consciousness level, $\phi(\Phi)$. This value, can in principle be computed for any information processing system, as long as the transition probability matrix between nodes of the system are known, and in essence is proportional to the amount of information gained by knowing the state of all nodes within the system versus having access to a limited purview of the system (Oizumi et al., 2014). Regrettably, computing this measure in complex biological systems is impossible from a practical standpoint because of its combinatorial search problem (but see Toker and Sommer, 2016, 2019, for interesting approaches circumventing current computing limitations).
In an effort to provide empirical evidence germane to theories of consciousness, we propose here simple neurophysiological benchmarks for consciousness as derived from the GNW and IIT, and test them empirically in single-unit recordings in nonhuman primates (see Materials and Methods).

\section{Materials and Methods}

Rationale, computation of integrated information ( $\Phi)$ and formalizing the role of multisensory integration in consciousness First we formalize the role of multisensory neurons that integrate information from multiple sensory modalities (operationalized as being driven by concurrent multisensory stimulation to a greater extent than by unisensory stimulation, "AND" gates) versus those that converge yet do not integrate (operationalized as responding to multiple sensory modalities but not to a greater extent in the multisensory condition, "XOR" gates = "OR" gates - AND gates; for an early characterization of multisensory neurons as Boolean gates, see Hartline et al., 1978). That is, we question whether under IIT mathematics it matters whether a neuron integrates its different sensory inputs, thus giving rise to a response that differs from the simple sum of these inputs (for a review of the seminal work first identifying true multisensory neurons where, for instance, audio + tactile $\neq$ audio-tactile see Stein and Stanford, 2008). Interestingly, IIT mathematics suggests that a simple three-node network (e.g., unisensory audio node, unisensory tactile node, and multisensory audiotactile node) merging on an integrative AND gate bears a greater degree of integrated information than one converging on an XOR gate $(\Phi=$ 0.78 vs $\Phi=0.25$, respectively; see Extended Data). Thus, according to this mathematical observation, it can be argued that as organisms' transition from consciousness to unconsciousness, neurons capable of integration should be those most readily impacted, hence providing us with the first testable prediction (Prediction 1). Second, again derived from IIT mathematics, it may be suggested that when organisms are conscious, neurons that integrate information should demonstrate neural properties that are present during consciousness to a greater degree than do neurons that simply convergent information (Prediction 2). Finally, according to GNW, neural ignition, indexed as singletrial coactivation, should be more readily apparent in conscious than unconscious states (Prediction 3).

To probe these predictions we simultaneously record single units from the primary somatosensory cortex $(\mathrm{S} 1)$ and ventral premotor cortex ( $\mathrm{vPM}$ ) of nonhuman primates as they were presented with audio, tactile, or audio-tactile stimuli. The monkeys were trained to report the presence of a stimulus (regardless of sensory modality) via button press to determine their trial-to-trial alertness during propofol-induced loss of consciousness (Ishizawa et al., 2016). We first characterize both the central (e.g., mean) and dispersion (e.g., variance) tendencies of multisensory responses in S1 and vPM neurons under normal wakefulness, and based on these responses, divide neurons into integrative or convergent categories. We then describe the impact of anesthesia on neuronal ascription to these categories as the animals lose consciousness (testing Prediction 1) and the degree to which they exhibit two neurophysiological indices that vary with consciousness; complexity (Lempel and Ziv, 1976) and noise correlations (Ecker et al., 2014; testing Prediction 2). Finally, the fact that recordings were performed simultaneously within a known microcircuit relevant to tactile subjective experience ( $\mathrm{S} 1$ and vPM; de Lafuente and Romo, 2006) allowed us to examine single-trial neural ignition as a function of consciousness (testing Prediction 3).

\section{Animal model}

Animals were handled according to the institutional standards of the National Institutes of Health and an approved protocol by the institutional animal care and use committee at the Massachusetts General Hospital. Two adult male monkeys (Macaca mulatta, 10-12 kg) were used.

\section{Behavioral task and experimental procedure}

The animals were trained in a behavioral task wherein following the onset of a start tone $(1000 \mathrm{~Hz}, 100 \mathrm{~ms})$ they were required to initiate each trial by holding down a button with their hand ipsilateral to the recording 
hemisphere. To successfully launch a trial (before loss of consciousness), the animals were required to hold the button within $1.5 \mathrm{~s}$ of the trial onset tone. Then, following button press, within a uniform random delay between 1 and $3 \mathrm{~s}$, one of four sensory stimulus sets was delivered ( $\mathrm{T}$, tactile air puffs; A, auditory stimuli; AT, simultaneous auditory and tactile; N, no stimuli). Air puffs during $\mathrm{T}$ trials were delivered at 12 psi to the lower part of the face contralateral to the recording hemisphere via a computercontrolled regulator with a solenoid valve (AirStim, San Diego Instruments). The eye area was avoided. Auditory stimuli during A trials were pure tones at $4000 \mathrm{~Hz}$ and at $80 \mathrm{~dB}$ SPL generated by a computer and delivered using two speakers $40 \mathrm{~cm}$ from the animal. Audio-tactile (AT) trials were simply the joint and simultaneous presentation of A and $\mathrm{T}$ trials. Catch trials $(\mathrm{N})$ were those when no stimulus was delivered. White noise (50 dB SPL) was applied throughout the trial to mask inherent noise derived from air puff and mechanical apparatus. All of the stimulus sets were presented randomly to the animal regardless of their behavioral response throughout the recording session. Following the presentation of the sensory stimulus the animals were required to keep holding the button down until the presentation of liquid reward (3 s post-stimuli onset). The monkeys were trained to perform a correct response on $>90 \%$ of the trials consistently for longer than $1.5 \mathrm{~h}$ in an alert condition. The animal's performance during the session was monitored and simultaneously recorded using a MATLAB-based behavior control system (Asaad and Eskandar, 2008a,b). Trial-by-trial behavioral responses were binned as a correct response (button holding until the trial end and release), failed attempt (early release, late touch, or no release of the button), or no response. Loss of consciousness was defined as the first no-response trial that was consistently followed by a lack of responses for the rest of anesthesia.

\section{Anesthesia}

Thirty minutes after initiating each recording session, propofol was infused for $60 \mathrm{~min}$ at a fixed rate $(200 \mathrm{~g} / \mathrm{kg} / \mathrm{min}$ for Monkey E; 230 or 270 $\mathrm{g} / \mathrm{kg} / \mathrm{min}$ for Monkey $\mathrm{H}$ ) through a vascular access port. The infusion rate of propofol was a priori determined to induce loss of consciousness in $\sim 10 \mathrm{~min}$ for each animal. No other sedatives or anesthetics were used during the experiment. The animal's heart rate and oxygen saturation were monitored continuously throughout the session (CANL-425SV-A Pulse Oximeter, Med Associates). The animals maintained $>94 \%$ oxygen saturation throughout the experiments.

\section{Neurophysiology data recording and preprocessing}

Before starting the study, a titanium headpost was surgically implanted on each of the two animals. A vascular access port was equally surgically implanted in the internal jugular vein (Model CP6, Access Technologies). Once the animals had mastered the behavioral task, extracellular microelectrode arrays (Floating Microelectrode Arrays, MicroProbes) were implanted into $\mathrm{S} 1$ and vPM through a craniotomy. Microelectrodes were also implanted in S2, but because of insufficient recorded neurons caused by a technical malfunction, here we focus our report on recordings from $\mathrm{S} 1$ and vPM. Each array $(1.95 \times 2.50 \mathrm{~mm})$ contained 16 platinum-iridium recording microelectrodes $(0.5 \mathrm{M} \Omega, 1.5-4.5 \mathrm{~mm}$ staggered length) separated by $400 \mu \mathrm{m}$. Landmarks on cortical surface and stereotaxic coordinates (Saleem and Logothetis, 2012) guided the placement of arrays. A total of five arrays were implanted in Monkey E (2 arrays in S1, 1 in S2, and 2 in vPM; all in the left hemisphere) and four arrays in Monkey H ( 2 arrays in S1, 1 in S2, and 1 and vPM; all in the right hemisphere). The recording experiments were performed after 2 weeks of recovery following the array surgery. All experiments were conducted in a radio frequency shielded recording enclosure.

Neural activity was recorded continuously and simultaneously from S1 and vPM through the microelectrode arrays while the animals were performing the behavioral task. Analog data were amplified, bandpass filtered between 0.5 and $8 \mathrm{kHz}$, and sampled at $40 \mathrm{kHz}$ (OmniPlex, Plexon). The spiking activity was obtained by high-pass filtering at 300 $\mathrm{kHz}$ and applying a minimum threshold of 3 SDs to exclude background noise from the raw voltage tracings on each channel. Subsequently all action potentials were sorted using waveform principal component anal- ysis (Offline Sorter, Plexon) and binned into 1 ms bins, effectively rendering the sampling rate $1 \mathrm{kHz}$.

\section{Neurophysiology data analyses}

Firing rate and Fano factor. Both central and dispersion tendencies of single-unit spiking activity in S1 and vPM were quantified as a function of stimulus modality as it is well established that mean firing rates alone do not fully characterize the properties of neural activity (Churchland et al., 2010). Regarding firing rates, spikes were first binned in $1 \mathrm{~ms}$ intervals, and epochs were centered on stimuli onset, ranging from $2000 \mathrm{~ms}$ before stimuli onset (i.e., $-2000 \mathrm{~ms}$ ), to $2000 \mathrm{~ms}$ after stimuli onset. Subsequently spike counts were effectuated within a $100 \mathrm{~ms}$ window, between $-500 \mathrm{~ms}$ and $1000 \mathrm{~ms}$, and in steps of $10 \mathrm{~ms}$. It must be noted that this analysis essentially low-passes time, and hence the exact timing of reported effects should not be emphasized. Analyses of firing rates were conducted on baseline-corrected rates. That is, every spike count function was centered along the $y$-axis (i.e., spikes/s) to zero according to their own baseline firing ( -500 to $0 \mathrm{~ms}$ post-stimuli onset). Spike counts were first averaged within a cell and across trials, and subsequently across neurons. In terms of statistical analyses, as the temporal dynamics of spiking activity was of interest, in particular within the GNW theory (Dehaene and Changeux, 2011; Dehaene et al., 2017) emphasizing sustained activity in aware and not unaware states, we conducted a timeresolved (at each $10 \mathrm{~ms}$ time point, 151 in total) 2 (State; Aware vs Unaware $) \times 2($ Area; S1 vs vPM $) \times 4$ (Stimulation; AT, T, A, N) independent samples ANOVA. The inter dependence of observations is difficult to ascertain within a neural network composed of neurons whose precise connections are unknown, and thus independent as opposed to dependent ANOVAs were conducted to adopt the most conservative approach (i.e., within-samples ANOVAs are statistically stronger than between-samples analyses). Similarly, to protect against type I error (i.e., false-positives) significant effects were only considered at $\alpha<0.01$ for at least three consecutive windows (i.e., 30 time points).

Regarding the intertrial variance in evoked responses associated with the distinct states of consciousness, stimuli modalities, and brain areas, Fano factors (FFs; i.e., ratio of spike-count variance to spike-count mean) were calculated (Fano, 1947). Statistical analysis is conducted as described for firing rates.

Neural index of multisensory integration. The hallmark for multisensory integration at the single-unit level is an evoked response to multisensory stimuli (e.g., AT) that may not be linearly predicted by responses to the constituent unisensory stimuli (e.g., A and T; Stein and Stanford, 2008). Thus, given the time-resolved results demonstrating sustained activity to sensory stimulation until $\sim 500 \mathrm{~ms}$ post-stimuli onset, mean spike counts to AT, T, A, and $\mathrm{N}$ trials were executed within this time period (Ghose et al., 2014). Subsequently, the (1) supra-additivity and (2) enhancement index of each neuron was computed (according to Eqs. 1 and 2, respectively). Historically, supra-additivity, the degree to which a multisensory response exceeds the sum of unisensory responses (Eq. 1), was considered the clearest indication of multisensory facilitation; nonetheless this feature is not as prominent in cortex as it is in subcortex (Kadunce et al., 1997; Stein and Stanford, 2008). Thus, we supplement the supra-additivity index with the enhancement index; the degree to which a multisensory response is greater than the maximal response to unisensory stimuli (Eq. 2). An enhancement index $>1$ indicates a neuron that is further driven by multisensory than unisensory stimulation. Supra-additivity (Eq. 1) and enhancement (Eq. 2) indices were computed as follows:

$$
\begin{aligned}
& \text { Supra-Additivity Index }=\frac{A T_{F R}}{\left(A_{F R}+T_{F R}\right)}, \\
& \text { Enhancement Index }=\frac{A T_{F R}}{\max \left(A_{F R}, T_{F R}\right)},
\end{aligned}
$$

where $A T_{F R}$ is the mean baseline-corrected firing rate for a particular neuron to audio-tactile stimulation, $T_{F R}$ is the mean baseline-corrected firing rate for the particular neuron to tactile stimulation, and finally $A_{F R}$ 
is the mean baseline-corrected firing rate for the particular neuron to auditory stimulation.

Categorization into convergent and integrative pools. Modeling results based on the IIT specify that a network converging on a neuron that integrates information (AND gate), as opposed to responding indiscriminately (XOR gate), ought to support a greater degree to consciousness. Hence, here we aim at testing two predictions that may follow from the IIT: (1) as an organism falls into unconsciousness, the neurons that are most impacted are those that integrate information (i.e., putatively anesthetics act on these neurons preferentially); and (2) neurons that integrate information exhibit the properties of consciousness when the organism is conscious. To test these predictions, we divided our population of neurons into those that integrate versus converge (see Fig. 4 and beyond). However, initially we simply describe the proportion of neurons that fit within each category (see Fig. 3 ) in a non-mutually exclusive fashion. A neuron that converges information is defined as a neuron that on average (i.e., across trials) responds, spike count from 0 to $500 \mathrm{~ms}$, to both unisensory auditory and tactile information beyond its baseline firing rate ( -500 to $0 \mathrm{~ms}$ ) plus $2 \mathrm{SD}$. That is, to qualify as convergent, the spiking count of a neuron to AT stimulation does not need to be examined. On the other hand, a neuron that integrates information is defined as a neuron that is most readily driven by the simultaneous presence of A and T information. Thus, neurons that respond to AT stimulation and do so to a greater degree than their maximal unisensory response (i.e., enhancement index $>1$ ) were initially classified as integrative. Importantly, beyond Figure 3 (e.g., to categorize the fate of neurons when the animal becomes unconscious and quantify neural complexity, noise correlations, and neural ignition) two mutually exclusive classes are created. Neurons that respond indiscriminately to sensory stimulation and not preferentially to multisensory versus unisensory presentations are classified as convergent, whereas those that exhibit multisensory enhancement without being considered convergent are taken to integrate information. Given the initial number of neurons in $\mathrm{S} 1$ and $\mathrm{vPM}$, this classification yielded a sufficient quantity of neurons exclusively categorized as convergent $(N=125)$ and integrative $(N=64)$ in S1, but not in vPM (convergent, $N=61$; integrative, $N=8$ ); thus, for the analyses specifically probing the difference between convergent and integrative neurons, analyses are restricted to S1. Further, given the heterogeneity of neuron's spike trains (see Figs. 1-3) for Figure 3 and beyond we considered a neuron as fitting within a particular category (e.g., A, T, AT convergent, AT integrative) if at some point between 0 and $1000 \mathrm{~ms}$ post-stimuli onset they met the particular criteria for at least 50 consecutive ms.

Equally of note, in Figure 3 neurons that are labeled to integrate auditory and tactile information (purple, orange, and green) are not first indexed for their unisensory responses. That is, although in S1, 49\% of neurons are classified as responding to a greater extent to AT stimulation than to the maximum unisensory stimulation, this latter unisensory response is not necessarily different from baseline activity. We consider this approach appropriate within the current aim of leveraging multisensory responses in querying consciousness theories, but it must be highlighted that multisensory enhancement may be more strictly considered to apply only when tactile, auditory, and audio-tactile responses are different from baseline, and the latter responses is greater than the maximal of the former two. Indeed, the categorization here is more in line with the recent emphasis within the study of multisensory integration to index covert multisensory processes (Bizley et al., 2007), in particular within classically considered primary sensory areas (Ghazanfar and Schroeder, 2006), than with the original description of multisensory integration in the late 1980s and early 1990s (Stein and Stanford, 2008).

Lempel-Ziv complexity. Categorizing the complexity of neural representations, operationalized as the number of distinct patterns present in data, has become of increasing popularity as of late in particular because of its ability to differentiate between states of consciousness given scalp electrophysiological data (Casali et al., 2013) and the belief that complexity is at least indirectly related to functional differentiation/integration, paramount notions with the IIT (Tononi et al., 2016). To quantify neural complexity, here we measure the Lempel-Ziv (LZ) complexity (Lempel and Ziv, 1976) associated with each spike train evoked as a consequence of AT, T, A, or N trials, and as a function of the animals' consciousness state. First, to equate entropy across conditions we converted spike trains into a continuous measure by convolving each trial with a Gaussian kernel with $\sigma=50 \mathrm{~ms}$, and then binarized each time-point within this trial by assigning a " 1 " to time points above the trial mean, and " 0 " to time points below the trial mean. Next, LZ was computed (Lempel and Ziv, 1976; for algorithm, see Amigó et al., 2004) in MATLAB within a sliding window moving between -500 and 750 ms post-stimuli onset, a length of $100 \mathrm{~ms}$, and step size of $50 \mathrm{~ms}$. Last, the same procedure was executed while randomly shuffling the binary sequence before calculating LZ. This shuffled LZ time-series represents a theoretical upper bound (i.e., random data has a higher LZ) and was used to normalize the calculated LZ from the non-shuffled data. Hence, a normalized LZ of 1 indicates "as complex as random noise", whereas lower values indicate the presence of structure in the data (Andrillon et al., 2016; Noel et al., 2018). Statistical analysis were conducted on non-corrected values to examine the basal complexity in spiking activity as a function of consciousness and whether neurons were categorized as convergent or integrative. Of note, the strongest evidence for the utility of Lempel-Ziv Complexity (LZc) in dissociating levels of consciousness comes from transcranial magnetic stimulation (TMS) evoked responses, which spatiotemporal pattern (i.e., time-series and EEG channels projected into voxels) is compressed and measured (Casali et al., 2013). Differently, here evoked activity is driven by sensory stimulation (Noel et al., 2018), and given the difficulty in knowing the precise location of neurons during extra-cellular recordings, we apply a temporal version of $\mathrm{LZc}$ (i.e., where there is no concatenation across space). This temporal-only version of $\mathrm{LZc}$ is that which has been applied to single units previously (Amigó et al., 2004), and has been shown to be elevated during psychedelic experiences (denoted LZs by Schartner et al., 2017).

Noise correlations. Although LZ complexity is arguably the most often used measure within the IIT framework (Tononi et al., 2016), it is not a traditional measure within neurophysiology. Thus, we sought to further probe the properties of convergent and integrative neurons, and their correspondence with the alteration in the particular measure as a function of consciousness state, with a neurophysiological measure that is well established to alter with consciousness state. Noise correlations (Ecker et al., 2014) express the amount of co-variability in the trial-totrial fluctuations of responses of two neurons to repeated presentations of the same stimuli, are central to questions of coding accuracy and efficiency (Averbeck et al., 2006), and are well established to be altered by consciousness state (Ecker et al., 2014). Thus, this measure was computed both in S1 and vPM neurons, as a function of consciousness state and stimuli modality. Noise correlations where computed as the Pearson correlation between all pairs of neurons recorded simultaneously within the same session (Ecker et al., 2014). Spike counts were effectuated for each trial on the $500 \mathrm{~ms}$ immediately following stimuli presentation (defined as the average time-period of neural response, and in concert with Ecker et al., 2014). We considered the noise correlation for a particular neuron its average correlation with all other neurons recorded in the same session.

Neural ignition. The GNW model points to the late amplification of relevant sensory activity, long-distance corticocortical synchronization at beta and gamma frequencies, and ignition of large-scale frontoparietal networks as neural measures of consciousness (Dehaene and Changeux, 2011). To test this prediction, we query at the single-trial level whether sensory stimulation leads to coactivation of both primary sensory areas (i.e., S1) and frontal regions (i.e., vPM) more commonly during conscious than unconscious states. For each neuron (both in S1 and vPM) we specify a threshold benchmarking reliable neural activity as the average spike count between -500 and 0 ms post-stimuli onset plus 2 SD. Similarly, the neural response is considered to be the spike-count between 0 and 500 post-stimuli onset. Then, iteratively we pick a neuron from S1 and a neuron from vPM and query whether on a particular trial did neither area respond, did solely S1 respond, did solely vPM respond, or did both S1 and vPM respond. A particular S1 neuron is subsequently paired with all neurons in $\mathrm{VPM}$ recorded during the same session, and finally its mean activation patterns (e.g., S1 and vPM active, vPM active, S1 active, or none) as a function of consciousness state and sensory stimulation are quantified. The same procedure is applied to vPM neu- 
rons. It must be highlighted that routinely mean firing rates are largely driven by strong responses in a few trials (Poison statistics; Churchland et al., 2010), and hence demanding a response within a particular trial to exceed baseline plus $2 \mathrm{SD}$ is a conservative approach yielding a great number of no-response trials. Nonparametric statistics are used in this analysis, as data did not confirm to the assumptions made by parametric inference statistics.

\section{Experimental design and statistical analyses}

The central point of the experimental design is that we present animals with unisensory (A or T) and multisensory (AT) cues to functionally label neurons as either integrative or convergent. Then these pools of neurons are rendered mutually exclusive, to assure statistical independence. Parametric inferential statistics are used when assumptions of these tests are met, and nonparametric are used if not (specific test indicated when introducing the result). This dataset has been used previously in another publication (Ishizawa et al., 2016) focusing on the local field potentials. Complementing the current analyses focused on single units and targeting specific theoretical arguments, Ishizawa et al., (2016) detail the network-level disruption of coherent beta oscillations between S1 and $\mathrm{vPM}$ preceding loss of consciousness, a transient increase in gam$\mathrm{ma} /$ beta band oscillations coincident with loss of consciousness, and the gradual ramp up of slow oscillations, first in S1 and then vPM, during and after loss of consciousness. The data are available from the corresponding author and the experiment was not preregistered.

\section{Results}

\section{Characterizing multisensory neurons in $\mathrm{S} 1$ and vPM}

The data, drawn as a subset of a previously published dataset (Ishizawa et al., 2016), comprise neural responses recorded from 293 single units in S1 (228 from Monkey E; 65 from Monkey $\mathrm{H}$ ) and 140 single units in vPM (87 from Monkey E; 53 from Monkey $\mathrm{H}$ ) recorded across 26 sessions (16 in Monkey E; 10 in Monkey H).

\section{Firing rates in $\mathrm{S} 1$ and vPM neurons}

Regarding the basic characterization of multisensory neurons in S1 and vPM, firing rates demonstrate (1) a reliable response to stimulus onset (Fig. 1, 1st and 3rd rows; colored horizontal bars indicate evoked response vs baseline at $p<0.01$ ); and (2) a reduction in activity when monkeys were rendered unconscious, both with regard to spontaneous (i.e., baseline, see Extended Data) and evoked activity (Fig. 1, 1st and 3rd rows; shaded area represents the difference between evoked activity when animals were conscious and not at $p<0.01$ ). Further, as expected given the known role of $\mathrm{vPM}$ in auditory processing (Lemus et al., 2009), neurons in vPM, but not $S 1$, generally responded to auditory stimulation (interaction at $p<0.01$ between 60 and $210 \mathrm{~ms}$ post-stimuli onset).

\section{Fano factors in S1 and VPM neurons}

FFs were calculated to assess intertrial response variability as a function of brain area, stimulation type, and state of consciousness. It has been previously reported that FFs are larger in an unconscious states $(\sim 2.2)$ than in conscious states (Ecker et al., 2014). Our results support a similar pattern, with FFs values being on average 1.45 under unconscious conditions and 1.16 under conscious conditions. This pattern fits nicely with the notion that conscious neural representations are more reproducible than unconscious ones (Schurger et al., 2010). The results also show an interesting pattern of variability changes as a function of stimulus onset. Whereas prior work has shown a reduction in variability upon stimulus onset (Churchland et al., 2010), the current results illustrate larger reductions in FF upon stimulus presentation in unconscious as opposed to conscious states (see Extended Data).

\section{Multisensory characteristics of S1 and vPM neurons}

Following the observation that neural responses to stimulus presentation were most robust during the $500 \mathrm{~ms}$ immediately following stimulus onset (see Extended Data), we performed a spike count during this interval to characterize the multisensory properties of neurons recorded (Ghose et al., 2014). The multisensory integrative responses of these neurons were quantified in two ways: relative to the sum of the unisensory responses (i.e., relative to the additive prediction; supra-additivity index), and (2) relative to the most effective unisensory response (i.e., quantifying the magnitude of the enhancement or depression of response; enhancement index; Murray and Wallace, 2012; Ghose et al., 2014). Here we report both indices for completeness, but operationally define integrative neurons as those with an enhancement index $>1$ (as both previous reports and the current dataset indicate that supra-additivity is rare in cortex (Sugihara et al., 2006; Bizley et al., 2007).

From the 293 single units recorded in S1, when the animals were aware two had a supra-additive index $>1$ (supra-additivity index $=1.23$ and 1.01; former depicted in Fig. 2A), whereas another two (different neurons) had supra-additive responses when the monkeys were rendered unconscious (supra-additivity index $=2.01$ and 1.01). Thus, supra-additivity is seemingly rare in S1. On the other hand, 100 neurons had enhancement indices $>1$ when the animals were conscious (Fig. $2 B$ ), a number that was reduced to 55 when the animals were rendered unconscious (25 of which indicating an enhancement index $>1$ in both aware and unaware states). Regarding vPM neurons, of the 140 neurons recorded, when the animals were aware two had a supra-additive index $>1$ (supra-additivity index $=1.03$ and 1.04), whereas only a single neuron was supra-additive when the animals were unconscious (supra-additivity index $=1.16$, distinct neurons in aware and unaware cases). Twenty-nine vPM neurons had enhancement indices $>1$ when animals were aware, a number that remained stable at 29 when monkeys were unconscious ( 2 of the 29 neurons were the same in aware and unaware states). Hence, multisensory supra-additivity appears equally infrequent in S1 as in $\mathrm{vPM}$, and interestingly there are seemingly more neurons demonstrating multisensory enhancement in S1 than vPM when animals were conscious ( $\mathrm{S} 1=34 \%$, vPM $=20 \%$ ) yet approximately equal proportions when the animals are unconscious $(\mathrm{S} 1=18 \%, \mathrm{vPM}=20 \%)$

The distributions of supra-additive and enhancement indices are well described by a Gaussian distribution, both when monkeys were conscious ( $\chi^{2}$ goodness-of-fit test, $p=0.28$ and $p=$ 0.24 , respectively) and unaware $(p=0.70$, and $p=0.90$, respectively; for a similar observation, see Bizley et al., 2007; Fig. 2C-F). A 2 (recording area; $\mathrm{S} 1$ vs $\mathrm{vPM}) \times 2$ (consciousness state; aware vs unaware) independent samples ANOVA on supra-additive indices (Fig. 2C,E) revealed main effects both of consciousness state $\left(F_{(1,862)}=5.9, p=0.015\right)$ and recording area $\left(F_{(1,862)}=11.05\right.$, $p<0.01)$, where supra-additivity indices were larger under aware $(\mathrm{M}=0.52, \mathrm{SEM}=0.006)$ than unaware $(\mathrm{M}=0.50, \mathrm{SEM}=$ $0.007)$ states, and larger in $\mathrm{S} 1(\mathrm{M}=0.52$, SEM $=0.006) \mathrm{com}-$ pared with vPM $(\mathrm{M}=0.48, \mathrm{SEM}=0.006)$. There was no interaction between these variables $\left(F_{(1,862)}<1, p=0.47\right)$. On the other hand, a similar analysis with regard to enhancement indices suggested no distinction between consciousness states $\left(F_{(1,862)}=\right.$ $2.45, p=0.11)$, no main effect of recording areas $\left(F_{(1,862)}=1.04\right.$, $p=0.30)$, and no interaction between these variables $\left(F_{(1,862)}=\right.$ 

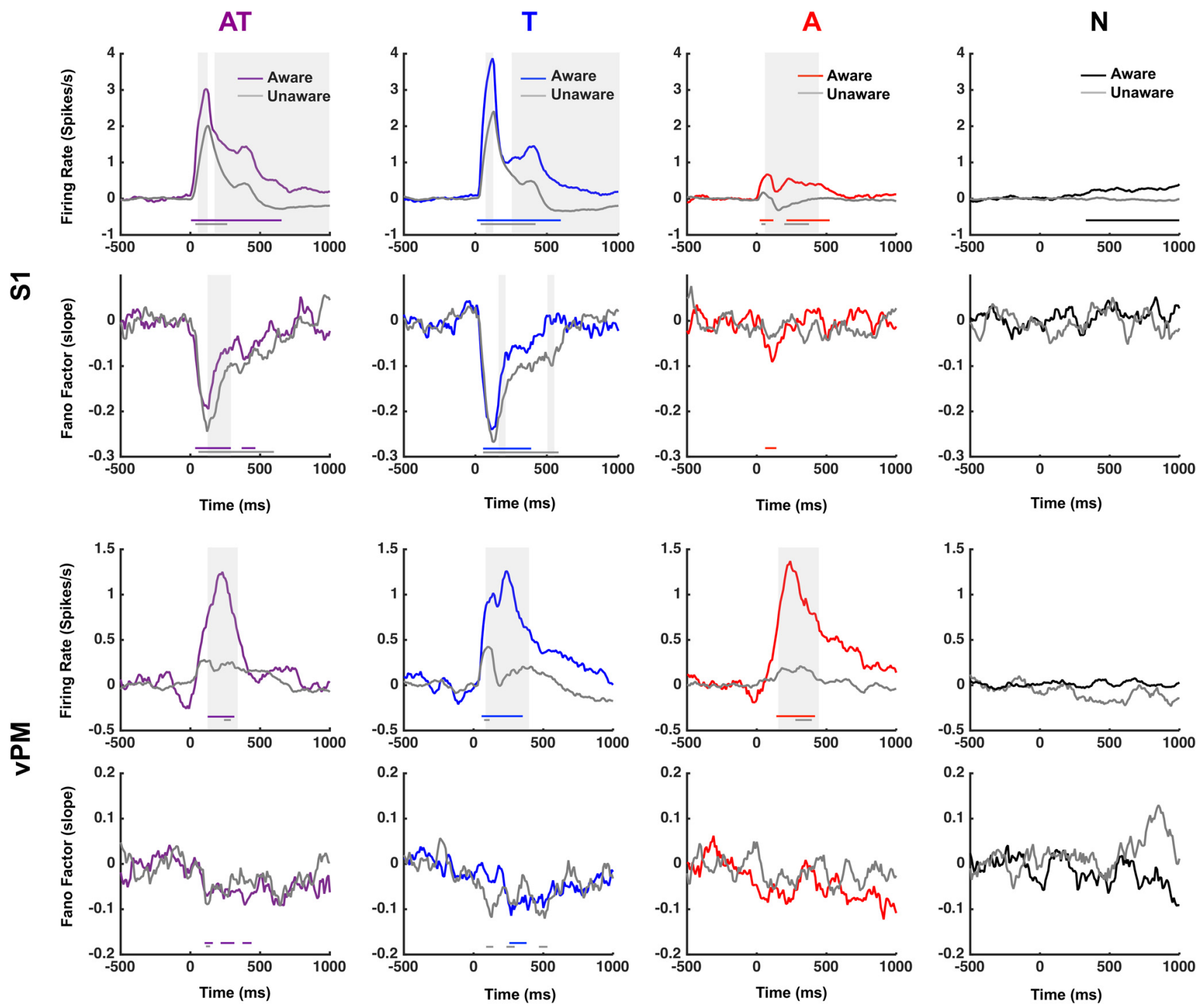

Figure 1. Time-resolved firing rates and FFs in $\mathrm{S} 1$ and vPM as a function of state of consciousness. Presentation of audio-tactile (AT; purple) and tactile (T; blue) stimuli evoked a reliable response in $\mathrm{S} 1$, while additionally the presentation of auditory ( $\mathrm{A}$; red) stimuli evoked a reliable response in vPM but not $\mathrm{S} 1$. Catch trials ( $\mathrm{N}$; black) did not evoked an increase in neural responses vis-à-vis baseline firing rate $(0, y$-axis). FFs were generally larger under states of unawareness than awareness (not depicted) and interestingly stimuli onset $(0, x$-axis) quenched variability in $\mathrm{S} 1$ (particularly onset of AT and T stimuli) but less $\mathrm{so}$ (and not differently between states of consciousness) in vPM. The time periods demonstrating a significant difference in evoked activity/FF as a function of state of consciousness (aware, colored; unaware, gray) are shaded in gray, whereas periods demonstrating a significant response vis-à-vis baseline are indicated by horizontal lines in each panel.

2.8, $p=0.09)$. These results highlight that the frequency and magnitude of multisensory integration may be dissociated (Olcese et al., 2013) and that supra-additive, where both unisensory responses are considered, and enhancement indices, where only the maximal unisensory response is compared with the multisensory response may provide very different views into integrative capacity. Further, the findings indicate a highly heterogeneous population. Taking the example of the enhancement indexes in S1 this metric indicates no overall change in the amount of integration at a population-level and across states of consciousness, yet examination of the classification of particular neurons reveals dramatic differences; shifting from 100 to 55 neurons in S1, only 25 of which were classified as integrating information both in aware and unaware states.

Fortunately within the current context aimed at examining theories of consciousness (e.g., IIT) we can leverage this variability to examine the outcome of neurons labeled as integrative or as convergent when animals are rendered unconscious. Figure 3 depicts the non-mutually exclusive compartmentalization of integrative and convergent neurons when monkeys were conscious. In this categorization scheme, neurons with an enhancement index $>1$ were considered to integrate information. In the conscious state, $43 \%$ of neurons in S1 respond to both audio and tactile stimuli, and thus can be categorized as convergent (Fig. 3, top left). For vPM, this value is $44 \%$ (Fig. 3, bottom left). When the categorization is done based on integrative criteria, $52 \%$ of neurons in S1 were found to integrate auditory and tactile information (i.e., respond to $\mathrm{AT}+(\mathrm{AT}>\max$ (A, T); Fig. 3, top right), whereas 33\% of neurons in $\mathrm{vPM}$ were categorized as integrative (Fig. 3, bottom right).

Importantly, to examine how this categorization is changed when animals are rendered unconscious (Prediction 1) and to quantify the extent to which they exhibit properties of consciousness (Prediction 2), we created mutually exclusive 
groups. Neurons that failed to respond to the auditory and tactile stimulus combination more that to the individual stimuli were classified as strictly convergent (convergent yet not integrative, or XOR gates). Conversely, neurons that responded more vigorously to the multisensory combination were labeled as integrative neurons, and were operationally categorized as AND gates. This classification of neurons into exclusive groups is important from a statistical perspective (in order not to create groups that are partially overlapping and overlapping to different extents across states of consciousness and recordings areas), and most importantly, from a theoretical perspective, creating AND and XOR neuronal pools. However, given the initial number of recorded neurons in S1 and vPM, this categorization scheme yielded a sufficient number of convergent $(N=125)$ and integrative $(N=64)$ neurons in S1, but not in vPM (convergent, $N=61$; integrative, $N=8$ ). Thus, for the analyses specifically probing the difference between convergent and integrative neurons in light of IIT (Predictions 1 and 2), analyses are restricted to S1.

Testing consciousness theory in multisensory neurons; information integration theory

Prediction 1: are integrative neurons most readily impacted by loss of consciousness? A first neurophysiological prediction that may be derived from the IIT is that network structured around an integrative neuron should lead to a greater degree of consciousness than one structured around a convergent neuron (see Extended Data). Hence, as an organism is rendered unconscious, the prediction is that integrative neurons should be most impacted.

As illustrated in Figure $4 A$, while a significant portion of S1 neurons labeled as convergent when the monkey was conscious became responsive exclusively to touch $(42.1 \%)$ following loss of consciousness, others were rendered nonresponsive $(24.1 \%)$ or transitioned to responding exclusively to auditory stimulation (2.5\%). $31.0 \%$ of neurons remained responsive to both auditory and tactile stimulation following loss of consciousness. On the other hand, of S1 neurons labeled as integrative when the animal was conscious, nearly two-thirds $(62.9 \%)$ remained integrative following the loss of consciousness. $18.6 \%$ of integrative neurons became exclusively responsive to tactile stimulation, $2.2 \%$ became exclusively responsive to auditory stimulation, and $16.3 \%$ became unresponsive. A $\chi^{2}$ test demonstrated that these proportions $(62.9 \%$ remaining as integrative but only $31.0 \%$ remaining as convergent) were significantly different from one another $(p=$ $0.001)$. Thus, and in contrast to the prediction derived from IIT, convergent neurons were more impacted when monkeys became unaware. It must be noted that this occurred despite the fact that arguably the requirements for being classified as "integrative" (i.e., responding to AT stimuli beyond their spontaneous and
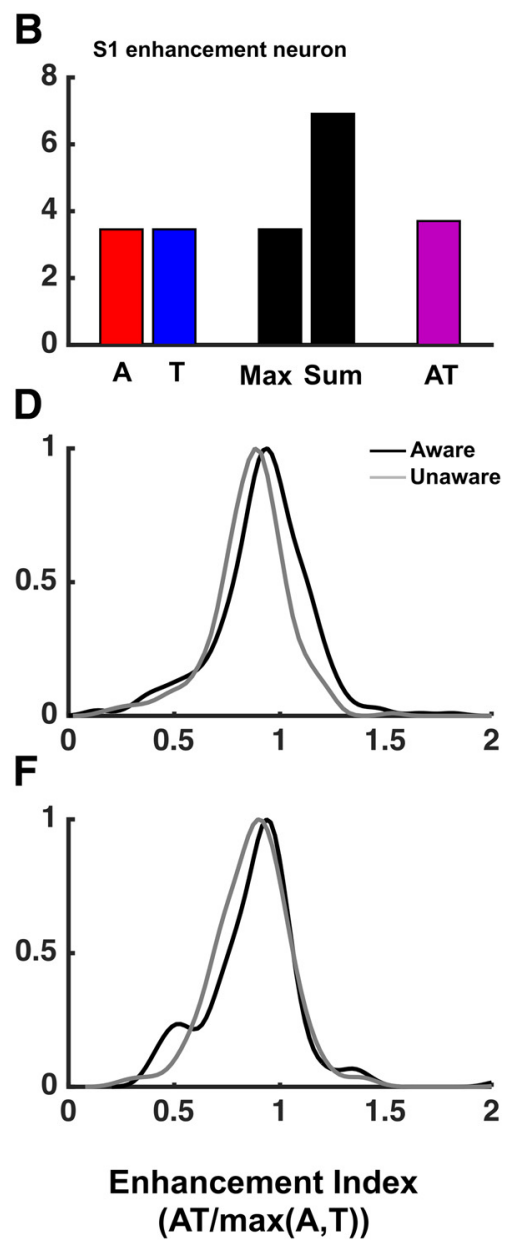

(AT/A+T)

\section{(AT/max(A,T))}

Figure 2. Characterizing multisensory neurons. A neuron whose multisensory response is greater than the sum of unisensory responses is said to be supra-additive $(\boldsymbol{A})$, while if it's greater than the greatest unisensory response it's considered to demonstrate ultisensory enhancement $(\boldsymbol{B}) . \boldsymbol{A}, \boldsymbol{B}$, Firing rates above a spontaneous rate (baseline-correction from -500 to $0 \mathrm{~ms} ; y$-axis $=0$ ) The distribution of supra-additive indices (left column) and enhancement indices (see Materials and Methods) were normally distributed both in S1 $(\boldsymbol{C}, \boldsymbol{D})$ and vPM $(\boldsymbol{E}, \boldsymbol{F})$, regardless of whether the animals were aware (black) or unaware (gray).

responding to AT stimuli beyond the maximal unisensory response) was more stringent than the bar required for a neuron to be classified as "convergent" (i.e., responding to A and T stimuli beyond their spontaneous firing rate).

We further examined whether these anesthesia-induced changes in neuronal responsiveness scaled with the degree to which neurons may be considered to be integrative. Although supra-additivity or multisensory enhancement are considered to be the hallmarks of multisensory integration, many multisensory neurons respond less vigorously to multisensory stimulation than their maximal response to unisensory stimulation (i.e., multisensory depression; Murray and Wallace, 2012). Nonetheless, these neurons are still considered to play an important role in multisensory integration (Kadunce et al., 1997). As illustrated in Figure $4 B$, although $56.4 \%$ of neurons exhibiting multisensory enhancement during consciousness had this enhancement preserved when the animal was rendered unconsciousness, only $36.8 \%$ of neurons that were categorized as exhibiting multisensory depression remained in that category upon the transition to unawareness. These proportions were significantly different from what is expected under the null distribution $(p=0.04)$. In sum, not only are integrative neurons not most readily impacted during the loss of consciousness, but the 
more a neuron is driven by paired stimulation toward response enhancement, the more likely it is to retain this enhancement during unconsciousness.

Prediction 2; do integrative neurons most readily demonstrate neural properties associated with consciousness?

In addition to probing the fate of convergent and integrative neurons as the animals were rendered unconscious, we also probed the degree to which these neurons exhibit neurophysiological properties associated with conscious states. The empirical measure most commonly associated with the IIT is the perturbation complexity index (PCI; Casali et al., 2013) and a component of this index, LZ complexity (Lempel and Ziv, 1976). In short, PCI is calculated by perturbing the cortex via TMS in an attempt to engage a distributed brain network, and subsequently compressing the spatiotemporal patterns of neural activity generated by the perturbation (using LZ) to measure the complexity of the response. In theory, the more distributed and recurrent the network, the larger should be the spatiotemporal complexity evoked by the perturbation. This empirical measure was directly derived from the IIT (Tononi et al., 2016) and has been shown to successfully differentiate between distinct levels of consciousness (Casali et al., 2013; Sarasso et al., 2015). In more simple applications, LZ has also been applied to resting state (Schartner et al., 2015, 2017) and stimulus evoked (Andrillon et al., 2016; Noel et al., 2018) neural activity (most commonly in scalp EEG datasets), and similar to PCI, has been shown capable of differentiating between levels of consciousness (Schartner et al., 2015, 2017). Here we first characterize time-resolved LZ complexity in spike trains as a function of consciousness state and modality of stimulation. After establishing predictions based on the state of consciousness in the neural population as a whole, we then classify S1 neurons into convergent or integrative pools, and examine which cohort most faithfully exhibits LZ complexity values that track the animal's consciousness state.

As illustrated in Figure 5A, overall LZ complexity was greater (across the entire epoch, see Extended Data) when monkeys were unaware (Fig. $5 A$, and Extended Data). This general property of LZ complexity was next indexed in convergent and integrative neurons. As depicted in Figure 5B, a 2 (consciousness state; aware vs unaware) $\times 2$ (neuron type; convergent vs integrative) ANOVA demonstrated a main effect of awareness (aware; $\mathrm{M}=$ $0.80, \mathrm{SEM}=0.001$; unaware; $\mathrm{M}=0.87, \mathrm{SEM}=0.002 ; p<0.01$, $\mathrm{df}$ between $=1$; df within $=188)$, yet no main effect of neuron type (all $p>0.11$ ). Most interestingly, however, there was a significant interaction between these variables $(p<0.01)$, as convergent neurons $(\mathrm{M}=0.79, \mathrm{SEM}=0.002)$ had marginally lower $\mathrm{LZ}$ complexity than integrative neurons $(\mathrm{M}=0.81$, SEM $=$ $0.002)$ when monkeys were aware $(p=0.052)$, yet this pattern

\section{Unisensory Responses and Multisensory Properties}
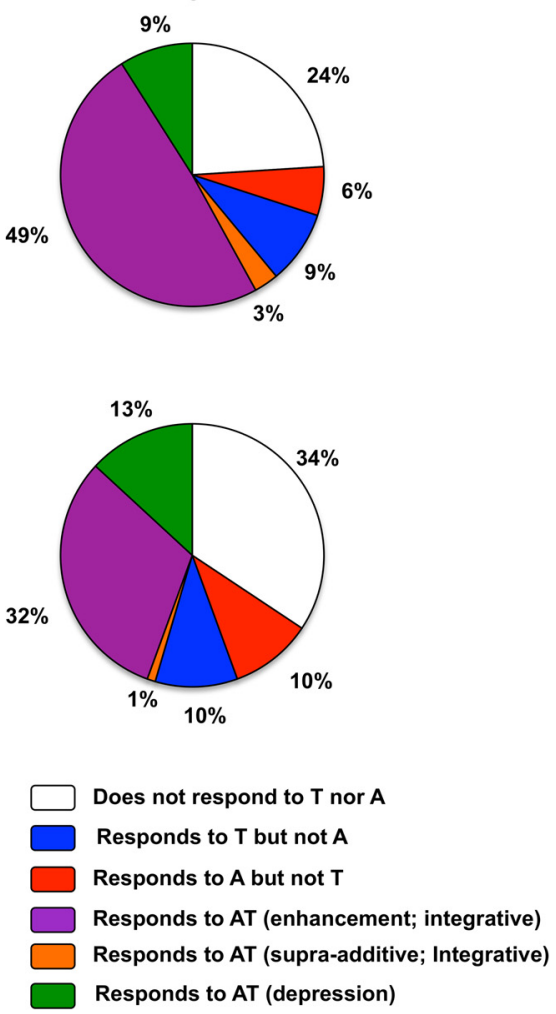

Figure 3. Non-mutually exclusive classification of neurons in S1 (top row) and vPM (bottom row) as convergent, integrative, unisensory, or nonresponsive. Left column, Neurons whose convolved firing rate excited their spontaneous rate plus 2 SD for at onsive or not (white). Next, if the neuron was responsive to AT stimulation, we queried whether during some epoch between (hest-stimuli onset their firing rate to AT stimulation was greater than the sum of A and T firing rates (supration but responded less to AT than to unisensory stimulation, the neuron was classified as demonstrating multisensory depression (green). Last, if they neuron did not respond to AT or A stimulation, but did to T, it was labeled as tactile (blue), whereas if a neuron did not respond to AT or T, but did to A, it was labeled as auditory (red).

reversed when monkeys loss consciousness (integrative; $\mathrm{M}=$ $0.86, \mathrm{SEM}=0.002$; convergent; $\mathrm{M}=0.88, \mathrm{SEM}=0.001, p=$ $0.045)$. Thus, convergent neurons tracked the state of consciousness, i.e., they exemplified the LZ behavior expected from a given state of consciousness (Fig. 5A), better than did integrative neurons.

These complexity results, just like the observed shifts in the distributions of convergent and integrative neurons following loss of consciousness, point in a direction counter to IIT; in that they suggest that convergent, as opposed to integrative, neurons more faithfully exhibit properties of consciousness. However, an important caveat is that there is relatively little empirical work quantifying LZ complexity in spike trains (Amigó et al., 2004; Abásolo et al., 2015). Hence, it may be useful to apply a similar logic, contrasting convergent and integrative neurons as a function of consciousness, while using a better-characterized neurophysiological measure within the context of consciousness studies. Thus, we next examined noise correlations.

Noise correlations have been shown to be strongly dependent upon state of awareness. For example, one study has demonstrated a sixfold increase in these correlations under an opioid 
A
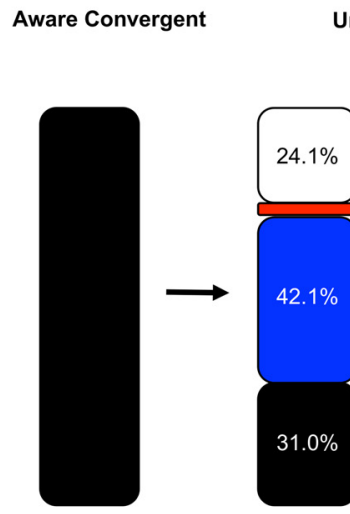

Unaware

Aware Integration

B

Aware Depression

Unaware
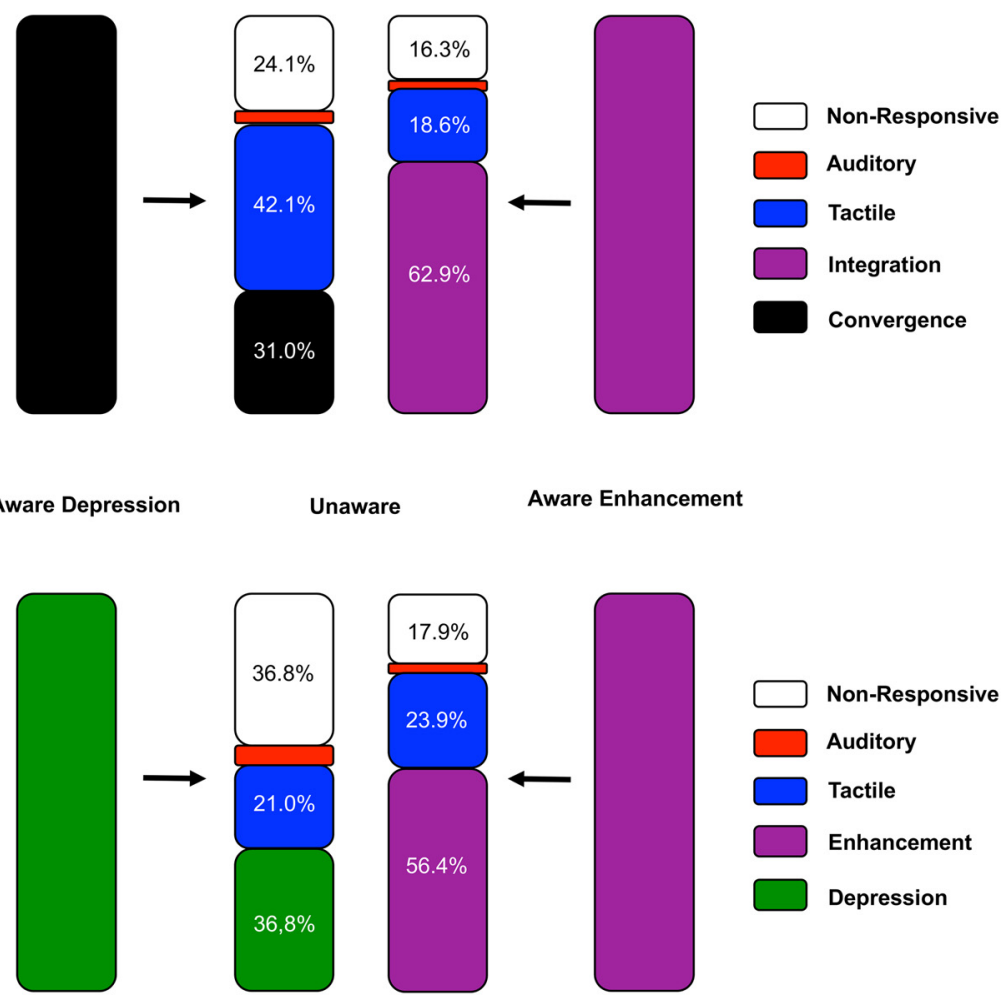

Figure 4. Transitions of $\mathrm{S} 1$ convergent and integrative neurons into distinct categories as monkeys are anesthetized. $\boldsymbol{A}$, The largest proportion of convergent neurons when monkeys were aware (black, leftmost) became responsive solely to tactile stimulation (blue) when monkeys were rendered unconscious (second column), whereas 31.0\% remained as convergent (black, second column). On the other hand, the majority of integrative neurons when monkeys were aware (rightmost column) remained as integrative (purple, third column). B, Similar to the contrast between convergent and integrative neurons, when contrasting neurons exhibiting multisensory depression (i.e., responds to AT but to a lesser extent than to unisensory stimulation) and enhancement (i.e., responds to AT and to a greater degree than to unisensory stimulation), results suggests that the larger the multisensory gain, the more neurons remain as integrative (vs not) when rendered unconscious. these variables $\left(F_{(1,187)}=29.63, p<\right.$ $0.001)$. The interaction seems to be driven by the fact that when monkeys were unconscious convergent neurons $(M=0.16$, SEM $=0.02$ ) showed a greater degree of noise correlations than integrative neurons $(\mathrm{M}=0.068, \mathrm{SEM}=0.008 ; p<0.001$; Fig. $6 B)$, and this difference disappeared during consciousness (convergent: $\mathrm{M}=$ 0.018, SEM $=0.004$; integrative: $\mathrm{M}=$ 0.028$, SEM $=0.008 ; p=0.067)$, significant $(p=0.067)$. Thus, although noise correlations were lower during conscious rather than unconscious states (in the population as a whole), this pattern is most readily apparent in convergent rather than integrative neurons.

Overall, the results suggest that consciousness is marked by a reduced degree of noise correlations, and that integrative neurons poorly track level of consciousness as indexed by this measure. In fact, these findings suggest a potential negative relationship between the degree of noise correlation that is typically associated with consciousness on one hand and with integration on the other. Namely, neurons that demonstrate the greatest degree of integration are those that show the greatest degree of noise correlation (in the conscious state). In support of this hypothesis, and as illustrated in Figure $6 B$ (middle and right), both the supraadditivity ( $r=0.15, p=0.02)$ and enhancement $(r=0.12, p=0.05)$ indices were positively correlated with the degree to which a neuron exhibited noise correlations. anesthetic compared with wakefulness (unaware $=0.05$; aware $=$ 0.008; Ecker et al., 2014). Accordingly, we examined noise correlations as a function of recording area ( $\mathrm{S} 1$ and $\mathrm{vPM})$, type of sensory stimulation (AT, T, A, and N), and consciousness state (aware and unaware). In addition to their relevance to the predictive framework laid out in the Rationale section (specifically Prediction 2), our assessment of noise correlations in our dataset has added importance as it represents the first measure of the impact of propofol on single-unit noise correlations in nonhuman primates.

As illustrated in Figure $6 A$, noise correlations demonstrated a striking increase from aware $(\mathrm{M}=0.02, \mathrm{SEM}=0.001)$ to unaware $(\mathrm{M}=0.11, \mathrm{SEM}=0.002)$ states $\left(F_{(1,3375)}=742.76, p<\right.$ $0.001)$. This effect was independent of recording area $\left(F_{(1,3375)}<\right.$ $1, p=0.86)$ and stimulation type $\left(F_{(1,3375)}=1.91, p=0.33\right)$, nor was there an interaction between variables in driving the degree to which noise correlated across single units (all $p>0.11$ ). Thus, the current dataset (using propofol) is in general agreement with the opioid-derived observation (Ecker et al., 2014) in that under anesthesia noise correlations increase by $\sim$ sixfold.

When restricting noise correlation analysis to the integrative and convergent neurons, we observed significant main effects of consciousness state $\left(F_{(1,187)}=91.56, p<0.001\right)$ and neuron type $\left(F_{(1,187)}=19.59, p<0.001\right)$, as well as an interaction between
Testing consciousness theory in multisensory circuits: GNW (Prediction 3)

Beyond the characterization of single-cell properties (i.e., firing rate, FF, complexity), it is possible to leverage the fact that neurons in both S1 and vPM, a well studied microcircuit involved in tactile subjective experience (de Lafuente and Romo, 2006), were concurrently recorded to test another prominent theory of consciousness; the GNW theory (Dehaene and Changeux, 2011). This theory states that sensory stimuli will elicit a conscious percept when the neural activity associated with the stimuli propagates through a broad frontoparietal network following neural ignition. Adapting this theoretical framework to the current experimental design, GNW predicts a higher likelihood of near concurrent neuronal firing in S1 and vPM (at the single-trial level) when animals are in a conscious state (and thus capable of conscious content) than when they are unconscious (Prediction 3).

To test this prediction we define a response threshold as exceeding spontaneous firing by 2 SDs (see Materials and Methods; the following results also hold defining a threshold of 1.0 or 1.5 $\mathrm{SD})$, and then calculate the percentage of trials that result in significant firing in $\mathrm{S} 1, \mathrm{vPM}$, or both $\mathrm{S} 1$ and $\mathrm{vPM}$, as a function of consciousness and sensory stimulation type. This approach yields relatively small percentages of trials cataloged as "active", which 


\section{A Lempel-Ziv Complexity}
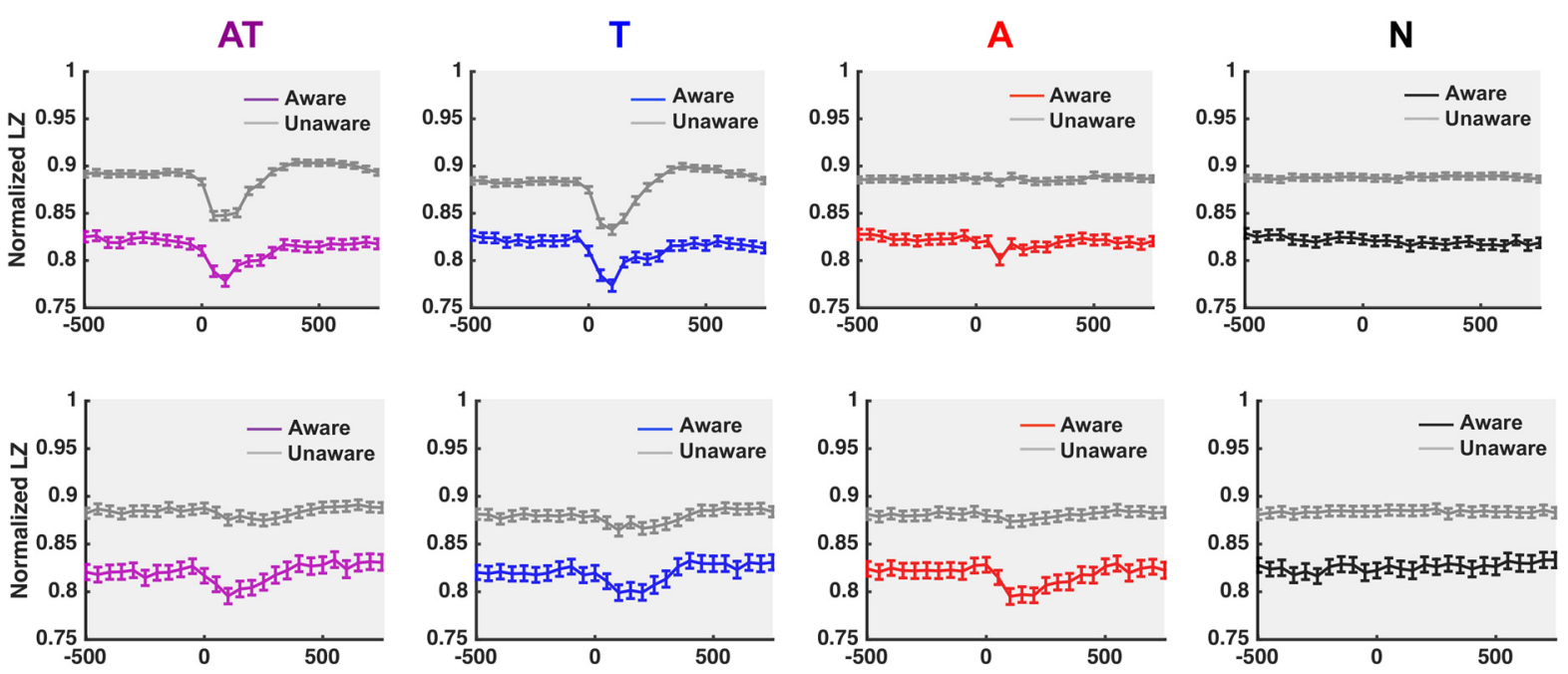

\section{B Lempel-Ziv Complexity as a Function of Neuron Type}

Aware

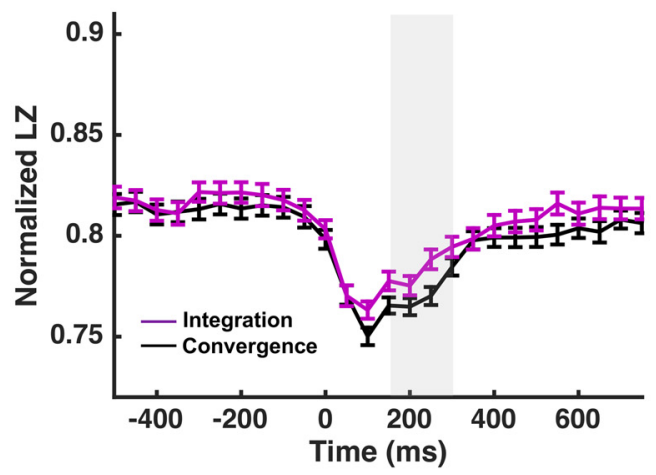

Unaware

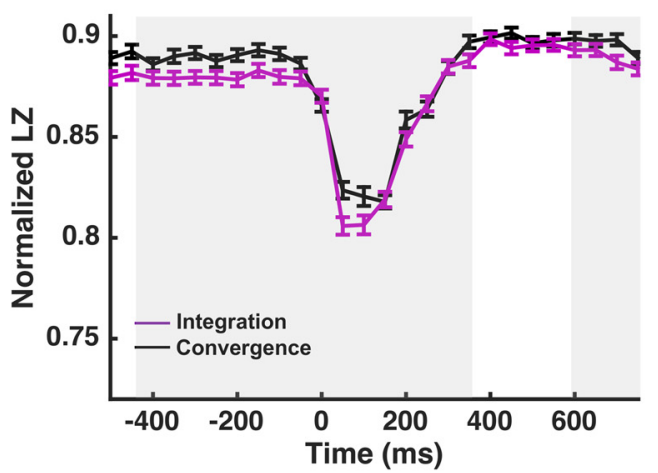

Figure 5. A, Time-resolved evoked Lempel-Ziv Complexity in spiking activity in S1 (top 2 rows) and vPM (bottom 2 rows) neurons as a function of consciousness state (aware, colored; unaware, gray) and sensory stimulation (AT, purple; T, blue; A, red; none, back). Most strikingly results suggest an increase in complexity ( $y$-axis) when monkeys were rendered unconscious. $B$, LZ in spiking activity in $\mathrm{S1}$ neurons as a function of consciousness state (aware, first column; unaware, second column) and whether the neuron was determined to converge (black) or integrate (purple) sensory information when aware. Results suggest that normalized LZ (top row, $y$-axis) is higher for integrative than convergent neurons when monkeys are aware (left column) yet this pattern reverses when monkeys are rendered unconscious (right column).

is to be expected given Poisson firing (i.e., the fact that on most trials the firing rates of most neurons change modestly, with relatively few neurons driving global population changes; Churchland et al., 2010), the high threshold set for labeling a trial as active, and the requirement for near concurrent firing. This approach, in other words, is statistically conservative. As highlighted in Figure 7 (left), results revealed that when animals were conscious, during combined AT stimulation, both S1 and vPM were concurrently active on $1.17 \%$ of trials (labeled "Concurrent Activation"). This number is reduced to $0.96 \%$ of trials during T stimulation, to $0.67 \%$ of trials during A stimulation, and to $0.28 \%$ of catch trials (main effect of stimulation type during awareness; Friedman test, $\chi^{2}=135, p<0.001$ ). The percentage of trials in which sensory stimulation resulted in the coactivation of S1 and vPM was significantly smaller when animals were rendered unconscious (main effect of consciousness state, Wilcoxon test, $Z=1135, p<0.001$ ) and did not differ across stimulation types (Friedman test during unawareness; $\chi^{2}=14.32, p=0.64$; stimulation type by consciousness state interaction, Friedman test of the difference between conscious vs unconscious as a function of sensory stimulation type, $\left.\chi^{2}=204.78, p<0.001\right)$. A similar pattern of results emerged when examining the number of trials that resulted in the independent activation of S1 and vPM (see Extended Data). Thus, in S1, 13.2\% of AT trials resulted in significant firing when monkeys were conscious, a number that was reduced to $10.5 \%$ in T trials (Wilcoxon, $p=1.61 \mathrm{e}-19$ ), and further reduced to $6.5 \%$ in $\mathrm{A}$ and $6.1 \%$ in $\mathrm{N}$ trials (T vs $\mathrm{A}, p=$ $1.28 \mathrm{e}-8$; A vs $\mathrm{N}, p=0.68$ ). In vPM, interestingly, the main effect of trial type in the conscious condition (see Extended Data) resulted from AT, T, and A all being different from $\mathrm{N}$ trials (all $p<$ $5.0 \mathrm{e}-20)$, as well as from vPM firing being most likely because of A stimulation $(\mathrm{M}=8.4 \%)$ than to $\mathrm{AT}(\mathrm{M}=7.4 \%)$ or $\mathrm{T}(\mathrm{M}=$ $7.5 \%$ ) stimulation (all $p$ values $<2.3 \mathrm{en} 5$ ). That is, activation of vPM was more probable because of A stimulation than T or AT stimulation; a stipulation that was not true (in fact opposite) in S1 or when examining coactivation of $\mathrm{S} 1$ and vPM. This finding 


\section{A Noise Correlation}

S1

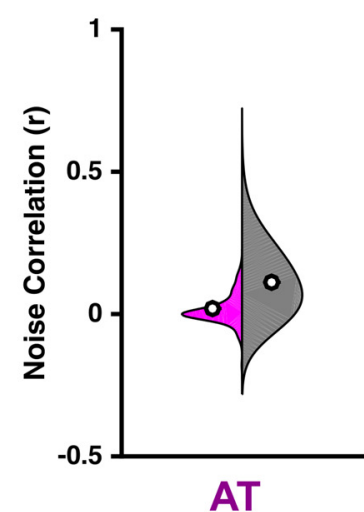

AT

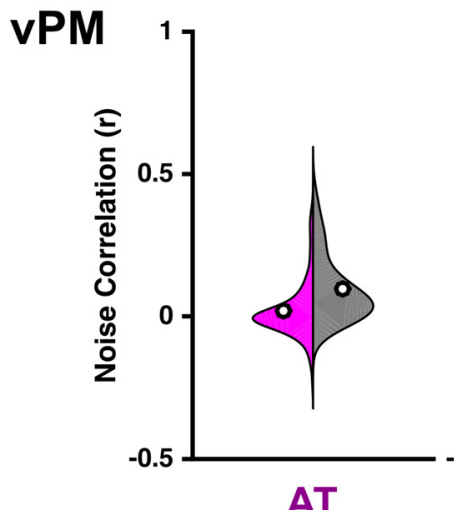

AT

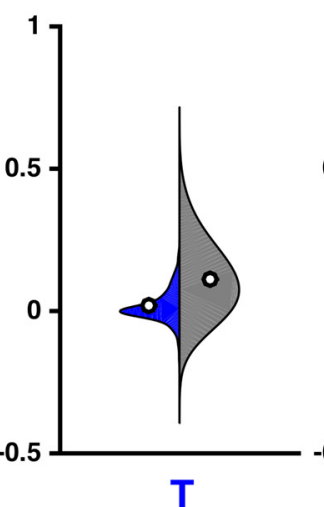

$\mathbf{T}$

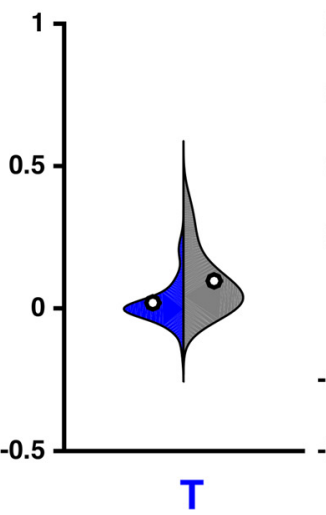

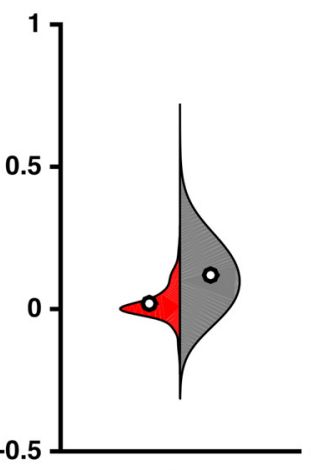

A

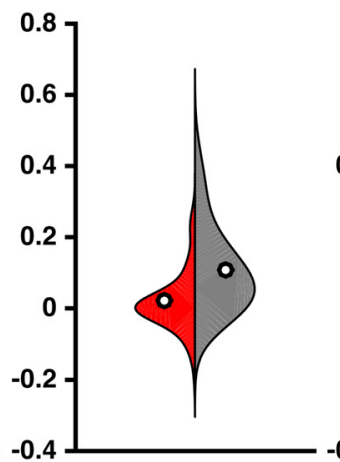

A
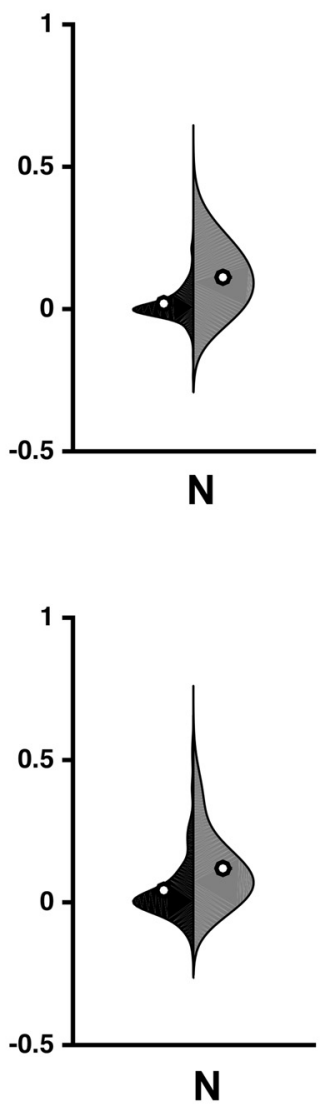

\section{B Noise Correlation as a Function of Neuron Type}
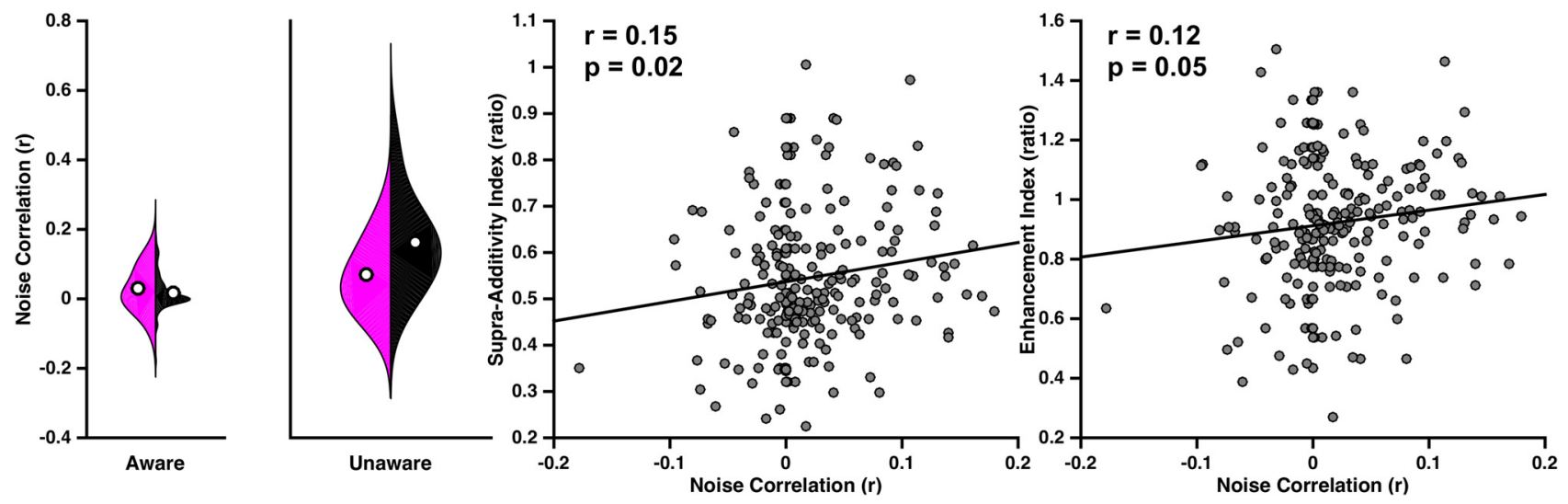

Figure 6. $\quad A$, Noise correlations in S1 (top) and vPM (bottom) as a function of consciousness state and sensory stimulation. Violin plots colored (purple, AT; blue, T; red, A; black, N) represent conscious states, while their gray counterparts illustrate noise correlations when the monkeys were rendered unconscious. White dots emphasize the mean. Overall, across all sensory modalities, noise correlations are sixfold greater under unconscious than conscious states. $\boldsymbol{B}$, Noise correlations in integrative and convergent $\$ 1$ neurons. When monkeys are aware (leftmost panel) integrative neurons (purple) exhibit a higher degree of noise correlations than neuron that integrate (black), whereas the contrary is true when monkeys were rendered unaware (second column). Further, when monkeys were aware, the more a neuron exhibited noise correlations (third and fourth panel, $x$-axis) the greater it's supra-additive (third panel, $y$-axis) and enhancement (fourth panel, $y$-axis) indices. White dots represent the mean of each distribution. 

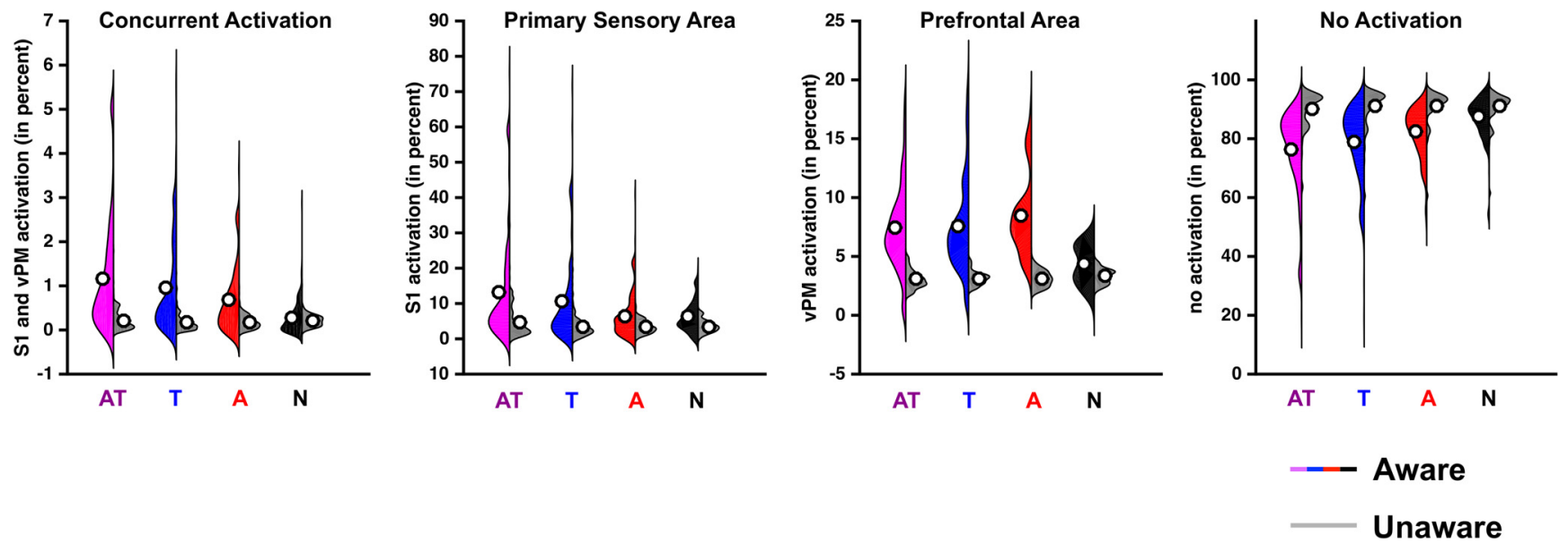

Figure 7. Percentage of trials that result in significant activation of $\mathrm{S1}, \mathrm{vPM}$, both or neither area, as a function of consciousness state and stimulation type. Concurrent activation is defined as the simultaneous activation of $\mathrm{S} 1$ and vPM (left). This phenomenon occurs to a greater degree when animals were conscious than unconscious, during AT (purple), $\mathrm{T}$ (blue), or A (red) stimulation, but not during catch trials (no stimulation). Second and third panels, respectively, demonstrate the number of trials that result in significant activation of S1 and vPM. Interestingly, although AT and T stimulation seemingly result in a greater percentage of trial demonstrating neural ignition and S1 activation than A stimulation, this is not the case for activation of vPM. Namely, auditory information seemingly reaches prefrontal areas via other routes. Last, rightmost panel illustrates the percentage of trials that do not result in significant activation; here the percentage is greater in unconscious than conscious trials, regardless of type of sensory stimulation. White dots represent the mean of each distribution.
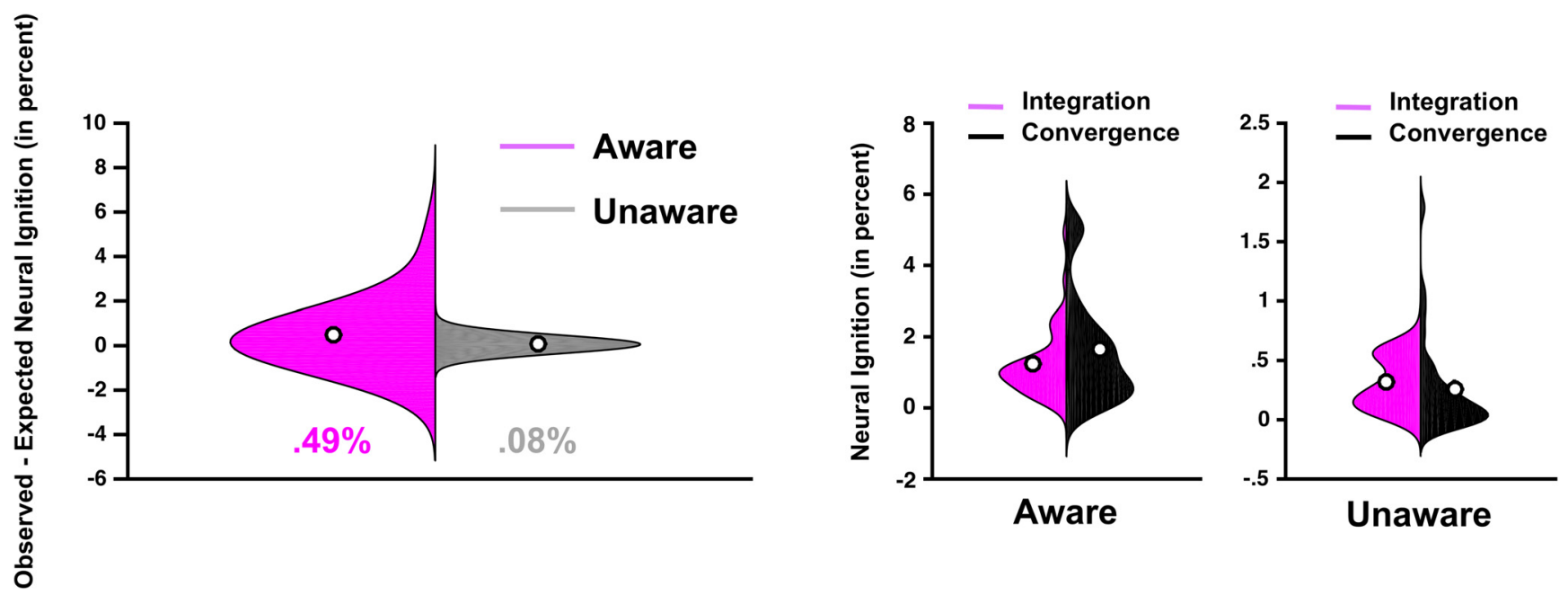

Figure 8. Neural ignition as a function of conscious state. Left, Difference in the observed percentage of trials resulting in neural ignition due to AT stimulation from the percentage of trials that would be predicted based on $S 1$ and vPM activation alone $(y=0)$, as a function of conscious state (aware, purple; unaware, gray). Right, Neural ignition due to AT stimulation in integrative (purple) and convergent (black) neurons as a function of consciousness state (aware, middle; unaware, right). White dots represent means of the distribution.

pinpoints that auditory information must arrive to $\mathrm{vPM}$ via a route that is not the same as how tactile information arrives in vPM (i.e., via S1), a finding that makes a great deal of sense because vPM is known to be part of the auditory "what" or ventral pathway (Rauschecker and Tian, 2000). Last, on the vast majority of trials sensory stimulation did not result in activity in either S1 or vPM, a finding that is most prominent in unconscious $(\mathrm{M}=91.0 \%)$ than conscious states $(\mathrm{M}=81.3 \%, Z=$ $37,949, p<0.001)$.

Thus, the overall pattern of results incorporating all cells recorded illustrate that when monkeys were conscious and sensory stimuli were being presented a greater number of trials resulted in coactivation of both primary sensory and "associative" areas than when animals were unconscious. The finding is in line with the GNW theory, but may represent a trivial result given that a larger number of trials also show exclusive activation in $\mathrm{S} 1$ or vPM when the animals were conscious. Hence, for pure probabilistic reasons, coactivation of S1 and vPM would be more likely under conscious than unconscious conditions. To address this concern, in a second step of analysis, we multiplied the likelihood of observing activation in S1 by the likelihood of observing activation in vPM and contrasted this predicted value to that observed (for both conscious and unconscious conditions). As shown in Figure 8, results demonstrated that in both the aware $(\mathrm{M}=0.49 \%$, one-sample $t$ test to zero, $p=$ $2.79 \mathrm{e}-22)$ and unaware $(\mathrm{M}=0.08 \%$, one-sample $t$ test to zero, $p=$ $2.95 \mathrm{e}-13$ ) cases, coactivation of S1 and vPM was more likely than what would be predicted by simply multiplying probabilities (Fig. 8; $y=0$ ). More importantly, the degree to which coactivation exceeded this prediction was greater under conscious conditions than under unconscious conditions $(t=6.2, p=6.41 \mathrm{e}-10$; for a recent demonstration that adding via photostimulation half a dozen spikes in a given neuron increases a downstream population activity by $0.5 \%$, similar to the effect size of consciousness here, see Chettih and Harvey, 2019).

Last, as the previous results (Figs. 5, 6; testing the IIT) had suggested that convergent neurons exhibited properties of con- 
sciousness to a greater degree than integrative ones, we sought to determine whether this was also true for these coactivation results. As illustrated in Figure 8 (middle and right) coactivation was generally more common when monkeys were conscious $(\mathrm{M}=1.5 \%)$ than unconscious $(\mathrm{M}=0.3 \%$, Mann-Whitney $U$, $p<0.001)$. Further, these likelihoods interacted with neuron type. Coactivation was not distinct in convergent and integrative neurons when the animals were aware (convergent, $\mathrm{M}=1.8 \%$; integrative, $\mathrm{M}=1.6 \%, p=0.37$ ). In contrast, convergent neurons demonstrated less coactivation than integrative neurons when the animals were aware (convergent, $\mathrm{M}=0.26 \%$; integrative, $\mathrm{M}=0.31 \%, p=0.004)$.

\section{Discussion}

Detailing the neural mechanisms enabling wakefulness and conscious experiences is a central question within systems neuroscience (Dehaene et al., 2017). As such, several theoretical frameworks have been put forth. Two influential frameworks are the IIT (Tononi et al., 2016) and GNW (Dehaene and Changeux, 2011). Unfortunately, these theories have seldom been tested neurophysiologically, and never within the same dataset. A series of concrete predictions can be generated from these theories, and in the current work we sought to generate such predictions and test them.

Starting from IIT mathematics, a strong prediction that can be made is that as an organism transitions from conscious to unconscious states, central integrative hubs (vs convergent hubs) of neural networks should be most impacted. Indeed, IIT states that the greater the information possessed by a network above and beyond its constituent parts, the more conscious the system (Tononi and Koch, 2015). Level of consciousness may be calculated and represented as the variable $\phi(\Phi)$. We demonstrate that within a simple three-node network, if the central node is an integrative (AND gate) node as opposed to a convergent (XOR gate) node, the value of $\Phi$ triples. In evaluating the neuronal data, we categorized neurons as either convergent or integrative and examined which class was most impacted by propofol administration. The assumption here is that cross-modal neurons in S1 and $\mathrm{vPM}$ receive information regarding the different senses from upstream areas, $\mathrm{S} 1$ and $\mathrm{VPM}$ in turn being the central node composed of AND and XOR functionality. Of course, this is an oversimplified biological neural network, but one that permits testing predictions derived from the IIT from a neurophysiological perspective. Contrary to our IIT-derived predictions, convergent, as opposed to integrative, neurons were most impacted by the administration of anesthesia. To further test predictions derived from IIT, we reasoned that when organisms were conscious, integrative neurons should exhibit neurophysiological properties of consciousness to a greater extent than convergent neurons (i.e., supporting lower $\phi$ values). The two measures chosen were LZ complexity and noise correlations, and we examined these as a function of stimulation type and conscious state. It is important to note that we do not aim to explain why neural complexity or noise correlations are altered by consciousness state, but simply to use these measures as "features of consciousness" and index how these properties are modulated in integrative versus convergent neurons as a function of consciousness. Complexity was chosen as it is the measure most often used within the IIT framework; though the relationship between $\Phi$, LZ complexity, TMSevoked complexity (PCI), and stimulus-evoked complexity is far from clear (Ibanez-Molina and Iglesias-Parro, 2018). The study of noise correlations was chosen as this measure has a stronger tradition within neurophysiology, and prior studies (Ecker et al.,
2014) have shown substantial increases in noise correlations after administration of an opioid anesthetic. Here again, findings indicated that convergent neurons most closely tracked the animals' consciousness state. Together, the findings of these neurophysiological analyses fail to provide strong empirical support for the IIT.

One of the novel findings of the current study is that under propofol, a $\mathrm{GABA}_{\mathrm{A}}$ potentiator (Brown et al., 2010), noise correlations are $\sim$ sixfold greater than during wakefulness. This finding is in line with previous single-unit recordings under a different anesthetic (opioid; Ecker et al., 2014) and concordant with a recent graph theory analysis of electrophysiological data showing that a change in local information processing efficiency, a measure that changes with noise correlations, could differentiate between distinct levels of responsiveness because of propofol administration (Lee et al., 2017).

The second theory tested was GNW (Dehaene and Changeux, 2011). In GNW the core concept is that during wakefulness a conscious experience should result in neural ignition; the broadcasting of sensory evidence throughout the brain. Concordant with the basic tenets of GNW, our results suggest that the coactivation of primary sensory areas and higher-order areas on a single trial is more likely under conscious than unconscious states. Importantly, the occurrence of this coactivation exceeded the expected values derived from the probability of noting S1 and vPM activations alone. These findings are concordant with a recent primate neuroimaging study demonstrating the breakdown of functional nodes (at the benefit of anatomically defined connections) during propofol, sevoflurane, and ketamine anesthesia (Uhrig et al., 2018), and a reduction in EEG directed functional connectivity across frontal and parietal areas during exposure to the same three drugs (Lee et al., 2013). Similarly, the seeming breakdown of frontoparietal/sensorimotor networks has been previously identified during propofol exposure in humans using electrocorticography (Malekmohammadi et al., 2018) and during ketamine exposure in nonhuman primates using multielectrode arrays (Schroeder et al., 2016), as in the current study (for further discussion of the effect of anesthetics on ignition in the context of GNW, particularly regarding interesting putative dose-dependent effects, see Mashour, 2018).

In addition to these coactivation findings, we analyzed firing rates in a time-resolved fashion, which allowed us the opportunity to see whether firing rates to sensory stimulation during consciousness were more sustained than during unconsciousness. As predicted by the GNW and well established in electroencephalography and electrocorticography (Del Cul et al., 2006, 2007; Gaillard et al., 2009), neural activity was more sustained when animals were conscious (vs unconscious). Together, our results provide empirical support (again through the lens of single neurons) for the GNW theory.

In addition to providing empirical neurophysiological evidence relevant to two theories of consciousness, our results make several novel contributions to the study of multisensory integration. First, to our knowledge, this is the first report to detail that supra-additivity and enhancement indices are normally distributed in both vPM and S1 of nonhuman primates (for a categorization of S1 neurons demonstrating enhancement and supra-additivity in rats, see Bieler et al., 2017). Second, we observed a large number of neurons exhibiting multisensory enhancement in S1 and vPM, yet very few that exhibited supraadditivity. These results comport well with the known multisensory convergence in vPM (Fogassi et al., 1996; Graziano et al., 1997), but also represent the first evidence that these neurons can 
integrate (in the classic supra-linearity definition; Stein and Stanford, 2008) this information. Third, we detail the dispersion tendencies associated with the firing patterns of (multi)sensory neurons in S1 and vPM. Variance in neuronal firing may be a result of a variety of causes (Churchland et al., 2010), both internal to the neuron or as a network property. Interestingly, although the FF is likely impacted by both these sources, an elevation in noise correlations likely reflects a source of comodulation. Thus, the current results demonstrating an increase in both FF and noise correlations during unconsciousness suggests a dynamical system in which the firing patterns of individual neurons are becoming more chaotic yet the population as a whole is more synchronously coactivated; an observation that is in line with reports suggesting a potentiation of slow oscillations and a reduction of high frequencies during unawareness (Ishizawa et al., 2016). Finally, from the observations that conscious states are seemingly associated with low noise correlations and that neurons showing multisensory convergence (as opposed to integration) more faithfully track consciousness according to this metric, we reasoned that perhaps a high degree of noise correlation is beneficial to multisensory integration. In fact, our results suggest a positive correlation between the amount a neuron shares noise with its neighbors, and the degree to which it exhibits multisensory integration. We find this result particularly interesting, as multisensory integration is a special form of integration; a form that has minimal shared variance at the sensory periphery, because information is transduced by different sensory organs. This relationship between shared noise and greater multisensory integration may be a result of larger dendritic arborizations in integrative neurons (Meredith et al., 2016, 2017), and may represent the neural instantiation of the postulation that stimulus correlation detection subserves the synthesis of information across the senses (Parise et al., 2012; Parise and Ernst, 2016, 2017).

In conclusion, we started from the IIT (Tononi et al., 2016) and the GNW (Dehaene and Changeux, 2011; Dehaene et al., 2017) to derive neurophysiological predictions relating to consciousness. We then leveraged multisensory neurons and circuits to functionally label neurons as convergent and integrative, and used these categorical distinctions to test IIT and GNW-derived predictions. The neurophysiological results generally support the GNW and not the IIT. However, important caveats exist. First, it is possible that the predictions we generated according to the IIT represented a higher bar to clear than those we generated from the GNW. Future work should aim at continuing to translate theoretical and philosophical postulates regarding consciousness- not only from IIT and GNW, but from some of the other theories as well (see Introduction for reference to other theories), into concrete and neurophysiologically testable hypotheses. Second, the IIT predictions derived and tested here represent the simplest implementation and interpretation of the theory possible. Only three nodes were used, and only a single node was changed in calculating different $\phi$ values. Further, we have assumed that single-unit spiking activity was a good approximation of the behavior of "nodes" within the IIT. This is far from trivial, as for example, in the IIT formalism nodes are either "on" or "off", yet real neurons can show graded levels of activity. Third, although simultaneously recording from $\mathrm{S} 1$ and vPM lends nicely to testing the GNW, given their known microcircuitry and their well established roles in tactile subjective experience (Romo et al., 2004; de Lafuente and Romo, 2006), it may be argued that these areas are not ideal for testing the IIT. Indeed, researchers supporting the IIT, recently suggest that anatomical correlates of consciousness are primarily localized to a "posterior hot zone". Thus, in the future it may be interesting to test similar ideas to those presented here in the posterior parietal cortex (or other cortical loci), or by leveraging novel large-scale electrophysiological techniques (Jun et al., 2017) while recording throughout the neocortical mantle (for arguments suggesting that the neural correlates of consciousness are in the "front" of the brain, see Odegaard et al. (2017)). Beyond specific objections related to the IIT, it must be emphasized that the results reported here are exclusive for propofol anesthesia. Hence, generalization of these results to the broader domain of consciousness must be done with caution. Future work may aim at replicating the above-described findings during the administration of several distinct anesthetics; the union of effects safely being able to be ascribed to consciousness. Further, it must be acknowledged that IIT is primarily a theory of consciousness-level, whereas GNW primarily focuses of consciousness-content. These different aspects of consciousness are closely related (as one does not consciously perceive the external environment if in an unconscious state), but dissociable. Here both aspects were conflated (i.e., it is assumed animals did not hear the auditory tone during unconscious-level), as the primary aim was to contrast IIT and GNW from a neurophysiological perspective. However, in the future it will be interesting to dissociate these dimensions. Last, the degree to which these findings generalize from macaques to humans is unknown. We can somewhat confidently assume that macaques have a subjective experience, given their similar behavioral repertoire to ours, but this remains a hypothesis. Regardless of model organism (macaque, human, or other), we consider that using sensory stimulation from distinct and multiple sensory modalities (e.g., Noel et al., 2015, 2018; Dykstra et al., 2017), as highlighted in the current report, may afford important leverage in the study of perceptual awareness and consciousness level by allowing functional characterization of neurons and neuronal ensembles.

\section{References}

Abásolo D, Simons S, Morgado da Silva R, Tononi G, Vyazovskiy VV (2015) Lempel-ziv complexity of cortical activity during sleep and waking in rats. J Neurophysiol 113:2742-2752.

Amigó JM, Szczepanski J, Wajnryb E, Sanchez-Vives MV (2004) Estimating the entropy rate of spike trains via lempel-ziv complexity. Neural Comput 16:717-736.

Andrillon T, Poulsen AT, Hansen LK, Léger D, Kouider S (2016) Neural markers of responsiveness to the environment in human sleep. J Neurosci 36:6583-6596.

Asaad WF, Eskandar EN (2008a) Achieving behavioral control with millisecond resolution in a high-level programming environment. J Neurosci Methods 173:235-240.

Asaad WF, Eskandar EN (2008b) A flexible software tool for temporallyprecise behavioral control in matlab. J Neurosci Methods 174:245-258.

Averbeck BB, Latham PE, Pouget A (2006) Neural correlations, population coding and computation. Nat Rev Neurosci 7:358-366.

Bieler M, Sieben K, Cichon N, Schildt S, Röder B, Hanganu-Opatz IL (2017) Rate and temporal coding convey multisensory information in primary sensory cortices. eNeuro 4:ENEURO.0037-17.2017.

Bizley JK, Nodal FR, Bajo VM, Nelken I, King AJ (2007) Physiological and anatomical evidence for multisensory interactions in auditory cortex. Cereb Cortex 17:2172-2189.

Block N, Carmel D, Fleming SM, Kentridge RW, Koch C, Lamme VA, Lau H, Rosenthal D (2014) Consciousness science: real progress and lingering misconceptions. Trends Cogn Sci 18:556-557.

Brown EN, Lydic R, Schiff ND (2010) General anesthesia, sleep, and coma. N Engl J Med 363:2638-2650.

Casali AG, Gosseries O, Rosanova M, Boly M, Sarasso S, Casali KR, Casarotto S, Bruno MA, Laureys S, Tononi G, Massimini M (2013) A theoretically based index of consciousness independent of sensory processing and behavior. Sci Transl Med 5:198ra105. 
Chettih SN, Harvey CD (2019) Single-neuron perturbations reveal featurespecific competition in V1. 173 Nature 567:334-340.

Churchland MM, Yu BM, Cunningham JP, Sugrue LP, Cohen MR, Corrado GS, Newsome WT, Clark AM, Hosseini P, Scott BB, Bradley DC, Smith MA, Kohn A, Movshon JA, Armstrong KM, Moore T, Chang SW, Snyder LH, Lisberger SG, Priebe NJ, et al. (2010) Stimulus onset quenches neural variability: a widespread cortical phenomenon. Nat Neurosci 13: 369-378.

Dehaene S, Changeux JP (2011) Experimental and theoretical approaches to conscious processing. Neuron 70:200-227.

Dehaene S, Lau H, Kouider S (2017) What is consciousness, and could machines have it? Science 358:486-492.

Del Cul A, Dehaene S, Leboyer M (2006) Preserved subliminal processing and impaired conscious access in schizophrenia. Arch Gen Psychiatry 63:1313-1323.

Del Cul A, Baillet S, Dehaene S (2007) Brain dynamics underlying the nonlinear threshold for access to consciousness. PLoS Biol 5:e260.

de Lafuente V, Romo R (2006) Neural correlate of subjective sensory experience gradually builds up across cortical areas. Proc Natl Acad Sci U S A 103:14266-14271.

Dykstra AR, Cariani PA, Gutschalk A (2017) A roadmap for the study of conscious audition and its neural basis. Philos Trans R Soc Lond B Biol Sci 372:20160103.

Ecker AS, Berens P, Cotton RJ, Subramaniyan M, Denfield GH, Cadwell CR, Smirnakis SM, Bethge M, Tolias AS (2014) State dependence of noise correlations in macaque primary visual cortex. Neuron 82:235-248.

Fano U (1947) Ionization yield of radiations: II. The fluctuations of the number of ions. Phys Rev 72:26-29.

Fogassi L, Gallese V, Fadiga L, Luppino G, Matelli M, Rizzolatti G (1996) Coding of peripersonal space in inferior premotor cortex (area F4). J Neurophysiol 76:141-157.

Gaillard R, Dehaene S, Adam C, Clémenceau S, Hasboun D, Baulac M, Cohen L, Naccache L (2009) Converging intracranial markers of conscious access. PLoS Biol 7:e61.

Ghazanfar AA, Schroeder CE (2006) Is neocortex essentially multisensory? Trends Cogn Sci 10:278-285.

Ghose D, Maier A, Nidiffer A, Wallace MT (2014) Multisensory response modulation in the superficial layers of the superior colliculus. J Neurosci 34:4332-4344.

Giacino JT, Fins JJ, Laureys S, Schiff ND (2014) Disorders of consciousness after acquired brain injury: the state of the science. Nat Rev Neurol 10: 99-114.

Graziano MS, Kastner S (2011) Human consciousness and its relationship to social neuroscience: a novel hypothesis. Cogn Neurosci 2:98-113.

Graziano MS, Hu XT, Gross CG (1997) Visuospatial properties of ventral premotor cortex. J Neurophysiol 77:2268-2292.

Hartline PH, Kass L, Loop MS (1978) Merging of modalities in the optic tectum: infrared and visual integration in rattlesnakes. Science 199:12251229.

Ibanez-Molina AJ, Iglesias-Parro S (2018) A comparison between theoretical and experimental measures of consciousness as integrated information in an anatomically based network of coupled oscillators. Complexity 2018:6101586.

Ishizawa Y, Ahmed OJ, Patel SR, Gale JT, Sierra-Mercado D, Brown EN, Eskandar EN (2016) Dynamics of propofol-induced loss of consciousness across primate neocortex. J Neurosci 36:7718-7726.

Joglekar MR, Mejias JF, Yang GR, Wang XJ (2018) Inter-areal balanced amplification enhances signal propagation in a large-scale circuit model of the primate cortex. Neuron 98:222-234.e8.

Jun JJ, Steinmetz NA, Siegle JH, Denman DJ, Bauza M, Barbarits B, Lee AK, Anastassiou CA, Andrei A, Aydın Ç, Barbic M, Blanche TJ, Bonin V, Couto J, Dutta B, Gratiy SL, Gutnisky DA, Häusser M, Karsh B, Ledochowitsch P, et al. (2017) Fully integrated silicon probes for highdensity recording of neural activity. Nature 551:232-236.

Kadunce DC, Vaughan JW, Wallace MT, Benedek G, Stein BE (1997) Mechanisms of within- and cross-modality suppression in the superior colliculus. J Neurophysiol 78:2834-2847.

Lamme VA (2006) Towards a true neural stance on consciousness. Trends Cogn Sci 10:494-501.

Lau H, Rosenthal D (2011) Empirical support for higher-order theories of conscious awareness. Trends Cogn Sci 15:365-373.
Laureys S, Owen AM, Schiff ND (2004) Brain function in coma, vegetative state, and related disorders. Lancet Neurol 3:537-546.

Lee M, Sanders RD, Yeom SK, Won DO, Seo KS, Kim HJ, Tononi G, Lee SW (2017) Network properties in transitions of consciousness during propofol-induced sedation. Sci Rep 7:16791.

Lee U, Ku S, Noh G, Baek S, Choi B, Mashour GA (2013) Disruption of frontal-parietal communication by ketamine, propofol, and sevoflurane. Anesthesiology 118:1264-1275.

Lempel A, Ziv J (1976) On the complexity of finite sequences. IEEE Trans Inf Theor 22:75-81.

Lemus L, Hernández A, Romo R (2009) Neural encoding of auditory discrimination in ventral premotor cortex. Proc Natl Acad Sci U S A 106 $14640-14645$.

Malekmohammadi M, AuYong N, Price CM, Tsolaki E, Hudson AE, Pouratian N (2018) Propofol-induced changes in $\alpha-\beta$ sensorimotor cortical connectivity. Anesthesiology 128:305-316.

Mashour GA (2018) The controversial correlates of consciousness. Science 360:493-494.

Meredith MA, Clemo HR, Corley SB, Chabot N, Lomber SG (2016) Cortical and thalamic connectivity of the auditory anterior ectosylvian cortex of early-deaf cats: implications for neural mechanisms of crossmodal plasticity. Hear Res 333:25-36.

Meredith MA, Clemo HR, Lomber SG (2017) Is territorial expansion a mechanism for crossmodal plasticity? Eur J Neurosci 45:1165-1176.

Monti MM, Vanhaudenhuyse A, Coleman MR, Boly M, Pickard JD, Tshibanda L, Owen AM, Laureys S (2010) Willful modulation of brain activity in disorders of consciousness. N Engl J Med 362:579-589.

Murray MM, Wallace MT (2012) The neural bases of multisensory processes. Boca Raton, FL: CRC.

Noel JP, Wallace M, Blake R (2015) Cognitive neuroscience: integration of sight and sound outside of awareness? Curr Biol 25:R157-R159.

Noel JP, Simon D, Thelen A, Maier A, Blake R, Wallace MT (2018) Probing electrophysiological indices of perceptual awareness across unisensory and multisensory modalities. J Cogn Neurosci 30:814-828.

Odegaard B, Knight RT, Lau H (2017) Should a few null findings falsify prefrontal theories of conscious perception? J Neurosci 37:9593-9602.

Oizumi M, Albantakis L, Tononi G (2014) From the phenomenology to the mechanisms of consciousness: integrated information theory 3.0. PLoS Comput Biol 10:e1003588.

Olcese U, Iurilli G, Medini P (2013) Cellular and synaptic architecture of multisensory integration in the mouse neocortex. Neuron 79:579-593.

Owen AM (2014) Disorders of consciousness: diagnostic accuracy of brain imaging in the vegetative state. Nat Rev Neurol 10:370-371.

Owen AM, Coleman MR, Boly M, Davis MH, Laureys S, Pickard JD (2006) Detecting awareness in the vegetative state. Science 313:1402.

Parise CV, Ernst MO (2016) Correlation detection as a general mechanism for multisensory integration. Nat Commun 7:11543.

Parise CV, Ernst MO (2017) Noise, multisensory integration, and previous response in perceptual disambiguation. PLoS Comput Biol 13:e1005546.

Parise CV, Spence C, Ernst MO (2012) When correlation implies causation in multisensory integration. Curr Biol 22:46-49.

Rauschecker JP, Tian B (2000) Mechanisms and streams for processing of "what" and "where" in auditory cortex. Proc Natl Acad Sci U S A 97: $11800-11806$.

Romo R, Hernández A, Zainos A (2004) Neuronal correlates of a perceptual decision in ventral premotor cortex. Neuron 41:165-173.

Saleem KS, Logothetis N (2012) A combined MRI and histology atlas of the rhesus monkey brain in stereotaxic coordinates, Ed 2. San Diego: Academic.

Sarasso S, Boly M, Napolitani M, Gosseries O, Charland-Verville V, Casarotto S, Rosanova M, Casali AG, Brichant JF, Boveroux P, Rex S, Tononi G, Laureys S, Massimini M (2015) Consciousness and complexity during unresponsiveness induced by propofol, xenon, and ketamine. Curr Biol 25:3099-3105.

Schartner M, Seth A, Noirhomme Q, Boly M, Bruno MA, Laureys S, Barrett A (2015) Complexity of multi-dimensional spontaneous EEG decreases during propofol induced general anaesthesia. PLoS One 10:e0133532.

Schartner MM, Carhart-Harris RL, Barrett AB, Seth AK, Muthukumaraswamy SD (2017) Increased spontaneous MEG signal diversity for psychoactive doses of ketamine, LSD and psilocybin. Sci Rep 7:46421.

Schroeder KE, Irwin ZT, Gaidica M, Nicole Bentley J, Patil PG, Mashour GA, Chestek CA (2016) Disruption of corticocortical information transfer 
during ketamine anesthesia in the primate brain. Neuroimage 134:459465.

Schurger A, Pereira F, Treisman A, Cohen JD (2010) Reproducibility distinguishes conscious from nonconscious neural representations. Science 327:97-99.

Stein BE, Stanford TR (2008) Multisensory integration: current issues from the perspective of the single neuron. Nat Rev Neurosci 9:255-266.

Sugihara T, Diltz MD, Averbeck BB, Romanski LM (2006) Integration of auditory and visual communication information in the primate ventrolateral prefrontal cortex. J Neurosci 26:11138-11147.

Toker D, Sommer F (2016) Moving past the minimum information partition: how to quickly and accurately calculate integrated information. arXiv:160501096.

Toker D, Sommer FT (2019) Information integration in large brain networks. PLoS Comput Biol 15:e1006807.
Tononi G (2012) Integrated information theory of consciousness: an updated account. Arch Ital Biol 150:293-329.

Tononi G, Edelman GM (1998) Consciousness and complexity. Science 282:1846-1851

Tononi G, Koch C (2015) Consciousness: here, there and everywhere? Philos Trans R Soc Lond B Biol Sci 370:20140167.

Tononi G, Boly M, Massimini M, Koch C (2016) Integrated information theory: from consciousness to its physical substrate. Nat Rev Neurosci $17: 450-461$.

Uhrig L, Sitt JD, Jacob A, Tasserie J, Barttfeld P, Dupont M, Dehaene S, Jarraya B (2018) Resting-state dynamics as a cortical signature of anesthesia inmonkeys. Anesthesiology 129:942-958.

van Vugt B, Dagnino B, Vartak D, Safaai H, Panzeri S, Dehaene S, Roelfsema PR (2018) The threshold for conscious report: signal loss and response bias in visual and frontal cortex. Science 360:537-542. 